\title{
SUPERCOOLING AND SUPERHEATING EFFECTS IN HETEROGENEOUS SYSTEMS*
}

\author{
BY \\ A. VISINTIN \\ Istituto di Analisi Numerica del C.N.R., Italy
}

\begin{abstract}
In the model for phase transitions in binary systems based on Fourier's and Fick's laws, the interface equilibrium condition $\theta=w$, relating the temperature $\theta$ and the chemical activity $w$, is here replaced by a relaxation dynamics for the liquid concentration $x$ :
\end{abstract}

$$
\frac{\partial \chi}{\partial t}+\tilde{H}^{-1}(\chi)=\beta(\theta, w) \quad\left(\tilde{H}^{-1}: \text { inverse of the Heaviside graph }\right) ;
$$

here $\beta \in C^{0}\left(\mathbf{R}^{2}\right)$ and $\operatorname{sign} \beta(\theta, w)=\operatorname{sign}(\theta-w)$. In the case of a single dimension of space, with an interface $x=s(t)$, a different dynamics can be considered:

$$
s^{\prime}(t)=\beta(\theta(s(t), t), w(s(t), t)) .
$$

Each of these laws is coupled with the energy conservation equation. Existence of at least one solution and maximum and minimum principles are proved for both problems. These results can be generalized in many ways, in particular, for temperature, concentration and phase-dependent coefficients. For the problem in a single space dimension, existence of a solution is proved also for the case of vanishing mass diffusivity in the solid. Uniqueness of the solution is shown for the problem in several dimensions of space.

1. Introduction and presentation of the models. 1 . The study of phase transitions in binary mixtures generalizes the classical Stefan problem. Its essential features are that the temperature, governed by the Fourier's equation, is coupled with the concentration, governed by the Fick's equation; the gradients of these variables fulfill Stefan-type discontinuity conditions at the interface $\mathscr{S}$, assumed to be a smooth surface. Actually in mass diffusion the concentration $c$ plays a role similar to that of the enthalpy in heat diffusion; in particular, $c$ is discontinuous across $\mathscr{S}$; the analog of the temperature $\theta$ is the chemical activity $w$, which is continuous across $\mathscr{S}$, as $\theta$ is.

\footnotetext{
* Received November 18, 1985.
} 
Under conditions of thermodynamic equilibrium, namely excluding supercooling and superheating effects, the phases are characterized as follows:

$$
\begin{cases}\theta<w & \text { in the solid phase } \\ \theta=w & \text { at the interface } \mathscr{S} \\ \theta>w & \text { in the liquid phase. }\end{cases}
$$

We denote by $\chi$ the characteristic function of the liquid phase (namely $\chi=0$ in the solid, $\chi=1$ in the liquid), and by $\tilde{H}$ the Heaviside graph (namely $\tilde{H}(\xi)=\{0\}$ if $\xi<0$, $\tilde{H}(0)=[0,1], \tilde{H}(\xi)=\{1\}$ if $\xi>0)$. (1.1) then yields

$$
\chi \in \tilde{H}(\theta-w) \text {. }
$$

In the framework of a weak formulation, the interface is not assumed to be a smooth surface, and mushy regions, namely mixtures of liquid and solid, are allowed; these correspond to zones where the liquid concentration $\chi$ attains values comprised between 0 and 1.

Problems of this sort have been studied by several authors $[2,6,7,8,12,13,19,20,21$, $30,31]$. Here we shall introduce two alternative models.

2. Phase transitions are driven by nonequilibrium conditions: "If the interface is not at the equilibrium temperature, then either melting or solidification occurs at a rate that increases with the difference between the actual temperature and the equilibrium temperature. For small departures from equilibrium the rate is approximately proportional to departure." (See [5], p.91.) We refer also to [15], Chapter 9.

As a first mathematical model of this physical picture, we propose a law of the form

$$
\frac{\partial \chi}{\partial t}+\tilde{H}^{-1}(\chi) \ni \beta(\theta, w),
$$

with $\beta \in C^{0}\left(\mathbf{R}^{2}\right)$ and $\operatorname{sign} \beta(\theta, w)=\operatorname{sign}(\theta-w)$. (1.3) is equivalent to the following variational inequality

$$
\left\{\begin{array}{l}
0 \leqslant \chi \leqslant 1 ; \quad \forall \xi \in[0,1], \\
{\left[\frac{\partial \chi}{\partial t}-\beta(\theta, w)\right] \cdot(\chi-\xi) \leqslant 0 .}
\end{array}\right.
$$

This represents relaxation towards the equilibrium condition (1.2) and introduces dynamical supercooled and superheated states, characterized by

$$
\theta<w \text { and } \chi=1, \quad \theta>w \text { and } \chi=0
$$

(respectively), contrasting with (1.1).

In the case of homogeneous systems the second member of (1.3) is replaced by a function depending just on $\theta$ :

$$
\frac{\partial \chi}{\partial t}+H^{-1}(\chi) \ni \tilde{\beta}(\theta),
$$

with $\tilde{\beta} \in C^{0}(\mathbb{R})$ and $\tilde{\beta}$ monotone in a neighborhood of $0 ;(1.3)^{\prime}$ is then coupled with an equation corresponding to the enthalpy balance.

This model was studied from a theoretical viewpont in [24]; an approximation procedure was proposed and an estimate of the order of convergence was then given in [23]. A physical justification of (1.3)' and some concrete applications were pointed out in [16]. 
3. In the case of a single dimension of space, a different model can be considered. Assuming that the interface is characterized by $x=s(t)$, the equilibrium condition becomes

$$
\theta(s(t), t)=w(s(t), t) .
$$

If $x<s(t)$ corresponds to the liquid and $x>s(t)$ to the solid, then one can also consider the following kinetic condition (see [3, pp. 222-223]):

$$
s^{\prime}(t)=\beta(\theta(s(t), t), w(s(t), t)),
$$

with $\beta$ as above. The corresponding problem for a homogeneous system was studied in [28]. An equation similar to (1.7) was studied by Crowley in [7] in modelling an annealing process used for the production of semi-conductor devices. There the phase transition is so fast that thermodynamic equilibrium cannot be assumed at the interface; moreover, the superheating is much smaller than the supercooling and consequently one is induced to consider the following kinetic condition:

$$
s^{\prime}(t)=\frac{1}{\varepsilon}(\theta-w)^{+}-a(\theta-w)^{-} \quad \text { on } \mathscr{S},
$$

with $a$ and $\varepsilon$ positive constants and $\varepsilon \ll 1 / a$.

In the case of several space dimensions, (1.7) should be replaced by

$$
\bar{v} \cdot \bar{n}=\left.\beta(\theta, w)\right|_{\mathscr{S}},
$$

where $\bar{v} \cdot \bar{n}$ is the normal component of the velocity of the phase transition front and is positive for melting. In several space dimensions it is natural to look for a weak formulation and to try to represent (1.9) in terms of $\chi$. Difficulties then arise in forcing $\chi$ to attain just the values 0 and 1 a.e. in $Q$; however, in the case of homogeneous systems, attempts in this direction have been made in [14, 25].

We notice that (1.3) corresponds to either the growth or the decrease of $\chi$ in the mushy region (see Fig. 1); this also yields a certain regularity for $\partial \chi / \partial t$. On the other hand, (1.7) and (1.9) represent the motion of a sharp interface $\mathscr{S}$ between the two phases (see Fig. 2). (1.3) ((1.7), respect.) is adequate when nucleation (crystal growth, respect.) is the dominant mechanism of phase transition.

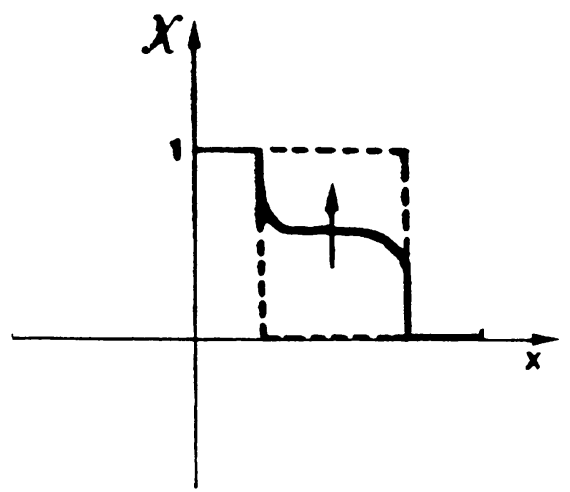

Fig. 1. 1st Mode of phase transition:

"Equiaxed Growth".

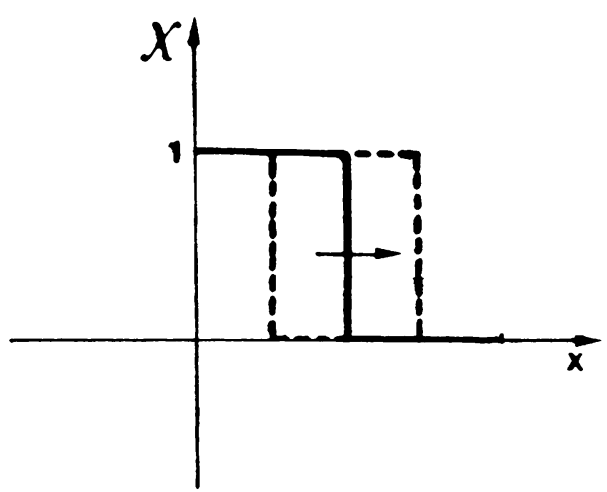

FIG 2. 2nd Mode of phase transition:

"Columnar growth". 
We stress that even in the one-dimensional case, (1.3) and (1.7) are not equivalent. This situation is quite different from the standard model for binary mixtures, in which the relaxation dynamics (1.3) and the kinetic law (1.7) are replaced by the corresponding equilibrium conditions (1.2) and (1.6), respectively. Indeed if equilibrium is assumed at the interface, then the strong formulation in terms of $(\theta, w, s)$ entails the weak formulation in terms of $(\theta, w, \chi)$, and also conversely under suitable regularity conditions [2, 8, 13]. This generalizes what happens for the standard Stefan problem.

4. In section 2 we present the physical problem. In sections 3 and 4 we study it in a single space dimension with the kinetic condition (1.7) and in several space dimensions with the relaxation law (1.3), respectively. In both cases we introduce variational formulations, prove existence and regularity results and also maximum and minimum principles. We stress that for the one-dimensional case, variational solutions are also strong solutions. In the case of several space dimensions, we also prove that $\chi \in W^{1, \infty}\left(0, T ; L^{2}(\Omega)\right) \cap$ $L^{\infty}\left(0, T ; H^{1}(\Omega)\right)$; that is, $\chi$ is much more regular than in the case with no phase relaxation.

Uniqueness of the solution is proved for the problem in several space dimensions of Section 4, whereas it is an open question for the one-dimensional problem of Section 3.

The previous results can be extended to more general physical models. Though we detail only the case of linearized phase diagrams (see figs. 6, 7 later on), our arguments hold also in the nonlinear case (see figs. 3, 5 later on). We stress that it is also possible to deal with temperature, concentration, and phase-dependent specific heat, as we discuss later on.

In the case of a single space dimension, we also study the asymptotic behavior as the mass diffusivity vanishes in the solid phase, as is physically reasonable. For instance, this assumption was made by Astarita and Sarti [1] in modelling certain phase transition phenomena in glassy polymers; there only the concentration evolution was considered and a kinetic law was assumed at the interface; this problem was also studied by Fasano, Meyer, and Primicerio in [11]. Our Theorem 3 can be applied also to phenomena of this sort, and yields the existence of a solution for the one-dimensional problem of coupled thermal and concentration evolution with no mass diffusion in the solid.

5. For a moment, let us consider linearized laws, with $\beta(\theta, w)$ replaced by $\mu(\theta-w), \mu$ being a positive constant. The choice between equilibrium and nonequilibrium conditions at the interface depends on the ratio between the relaxation coefficient $\mu^{-1}$ and the characteristic time-scale of the phenomenon under consideration. From a more mathematical viewpoint, evolution laws of the type (1.3) and (1.7) are well-fitted for proving existence results and yield extra-regularity properties for the solution. On the contrary, for the problems corresponding to the equilibrium conditions (1.2) and (1.6), in general even the existence of a solution is not evident.

An important open question concerns the asymptotic behavior as the phase relaxation time $\mu^{-1}$ vanishes; do then the solutions, or suitable subsequences, converge to a solution of the reduced problem, in which (1.3) and (1.7) are replaced by (1.2) and (1.6) (respectively)? An affirmative answer can be given for one-component systems [28]. A similar question arises as $\varepsilon \rightarrow 0$ in (1.8); in this case the numerical results obtained by Crowley in [7] seem to indicate the convergence. 
6. A different approach to phase transitions in heterogeneous systems is based on the so-called "nonequilibrium thermodynamics". Mathematical studies based on this model were started by Donnelly in [9] and continued by Luckhaus and the present author in [18]. In [24] a relaxation dynamics for the phase variable $\chi$ was introduced into such a model.

Finally, let us shortly consider the problem of coupled heat and electric evolution in a two-phase electric conductor; here the temperature is coupled with the electro-magnetic variables governed by Maxwell's equations. The mathematical structure of this problem is quite similar to that of the standard formulation of the binary alloy problem considered here. Nonequilibrium interface conditions and supercooled and superheated states can be introduced also here and lead to mathematical developments similar to those of the present paper. Also, for this problem, an alternative approach based on the "nonequilibrium thermodynamics" was studied in [29].

The results of the present paper were announced in [27].

2. The physical problem. 1 . We consider a mixture of two components, which are completely soluble in each other in all proportions in both the liquid and solid states. We denote the temperature by $\theta$ and the concentration of one of the two components by $c$ $(\in[0,1])$. We assume that the system occupies a bounded domain $\Omega \subset \mathbf{R}^{N}(N \geqslant 1)$ in a time interval $[0, T](T>0)$; we denote by $Q_{1}$ and $Q_{0}$ the space-time domains corresponding to the liquid and solid phases, respectively, and by $\mathscr{S}$ the interface between them.

We shall deal with a simplified model, in which, in particular, convection and crosseffects between heat and mass diffusion are neglected. Then Fourier's and Fick's laws have the form:

$$
\begin{gathered}
\gamma_{1, i} \frac{\partial \theta_{i}}{\partial t}-\nabla \cdot\left(\overline{\bar{k}}_{1, i} \cdot \nabla \theta_{i}\right)=0 \quad \text { in } Q_{i} \quad(i=0,1), \\
\frac{\partial c_{i}}{\partial t}-\nabla \cdot\left(\tilde{\bar{k}}_{2, i} \cdot \nabla c_{i}\right)=0 \quad \text { in } Q_{i} \quad(i=0,1) ;
\end{gathered}
$$

in each phase, $\gamma_{1, i}(>0)$ is the specific heat multiplied by the density, $\overline{\bar{k}}_{1, i}(>0)$ is the thermal conductivity tensor, $\widetilde{\bar{k}}_{2, i}(>0)$ is the mass conductivity tensor; $\nabla:=\left(\frac{\partial}{\partial x_{1}}, \ldots, \frac{\partial}{\partial x_{N}}\right)$.

The balances of heat and mass at the interface yield

$$
\begin{gathered}
\left(\overline{\bar{k}}_{1,1} \cdot \bar{\nabla} \theta_{1}-\overline{\bar{k}}_{1,0} \cdot \bar{\nabla} \theta_{2}\right) \cdot \bar{n}=-L \bar{v} \cdot \bar{n} \quad \text { on } \mathscr{S}, \\
\left(\tilde{\bar{k}}_{2,1} \cdot \bar{\nabla} c_{1}-\tilde{\bar{k}}_{2,0} \cdot \bar{\nabla} c_{2}\right) \cdot \bar{n}=-\left(c_{1}-c_{0}\right) \bar{v} \cdot \bar{n} \quad \text { on } \mathscr{S} ;
\end{gathered}
$$

here $L$ is the latent heat of phase transition multiplied by the density; $\bar{v}$ is the velocity of the solidification front; $\bar{n} \in \mathbf{R}^{N}$ is normal to $\mathscr{S}(t):=\mathscr{S} \cap\left(\mathbf{R}^{n} \times\{t\}\right) ; \theta_{i}$ and $c_{i}$ are the limits of $\theta$ and $c$ from $Q_{i}$ onto $\mathscr{S}(i=0,1)$.

As we said, we assume that the two components of the system are soluble in each other in all proportions in both the liquid and solid states; this leads to an equilibrium diagram like Fig. 3. The situation is more complicated when the components are soluble only in a bounded range of concentrations in the solid state $[5,15]$, a situation we shall not address 


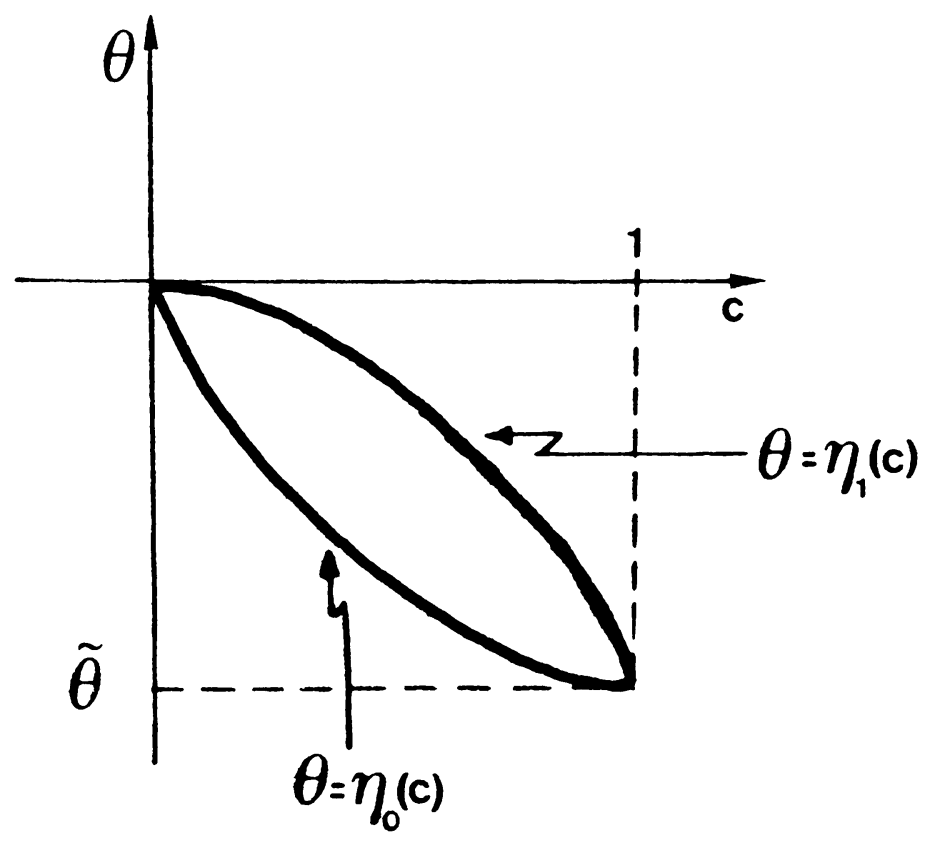

FIG. 3. Phase diagram for a mixture of two components, soluble in each other in all proportions in both the liquid and solid state.

here. In our case the thermodynamic equilibrium corresponds to the following conditions at the interface:

$$
\begin{gathered}
\theta_{1}=\theta_{0} \quad \text { on } \mathscr{S}, \\
\theta_{i}=\eta_{i}\left(c_{i}\right) \quad \text { on } \mathscr{S} \quad(i=0,1),
\end{gathered}
$$

where $\eta_{i} \in C^{1}([0,1]), \eta_{1} \geqslant \eta_{0}, \eta_{1}(0)=\eta_{0}(0)=0, \eta_{1}(1)=\eta_{0}(1)=\tilde{\theta}$ (with $\tilde{\theta}<0$, say).

If supercooled and superheated states are excluded, we have

$$
\theta \geqslant \eta_{1}(c) \text { in } Q_{1}, \quad \theta \leqslant \eta_{0}(c) \text { in } Q_{0} .
$$

Following a standard technique (see $[8,13]$ e.g.), we introduce the function

$$
w:=\eta_{i}(c) \quad \text { in } Q_{i} \quad(i=0,1),
$$

which physically represents the chemical activity; thus $\tilde{\theta} \leqslant w \leqslant 0$ in $Q$. Then (2.5), , (2.7) yield

$$
\begin{gathered}
w_{1}=w_{0}=\theta \quad \text { on } \mathscr{S}, \\
\theta \geqslant w \quad \text { in } Q_{1}, \quad \theta \leqslant w \quad \text { in } Q_{0} .
\end{gathered}
$$

Hence, still denoting by $\chi$ the characteristic function of the liquid phase and by $\tilde{H}$ the Heaviside graph, we have

$$
\chi \in \tilde{H}(\theta-w) \quad \text { in } Q
$$

We set

$$
v=-c \quad \text { in } Q,
$$




$$
\overline{\bar{k}}_{2, i}:=\frac{\overline{\bar{k}}_{2, i}}{\eta_{i}^{\prime}\left(\eta_{i}^{-1}(w)\right)} \quad \text { in } Q_{i} \quad(i=0,1) .
$$

For the moment we assume that the coefficients $\gamma_{1, i}, \overline{\bar{k}}_{1, i}$, and $\cong_{\bar{k}}$ are constant and phase-independent, and set

$$
\gamma_{1}:=\gamma_{1,1}=\gamma_{1,0}, \quad k_{j}:=k_{j, 1}=k_{j, 0} \quad(j=1,2)
$$

later on we shall remove these restrictions.

The previous setting corresponds to the following system:

$$
\begin{aligned}
\frac{\partial u}{\partial t}-\bar{\nabla} \cdot\left(\overline{\bar{k}}_{1} \cdot \bar{\nabla} \theta\right)=0 & \text { in } \mathscr{D}^{\prime}(Q), \\
\frac{\partial v}{\partial t}-\bar{\nabla} \cdot\left(\overline{\bar{k}}_{2} \cdot \bar{\nabla} w\right)=0 & \text { in } \mathscr{D}^{\prime}(Q), \\
u=K_{\chi}(\theta) & \text { in } Q, \\
v=G_{\chi}(w) & \text { in } Q,
\end{aligned}
$$

where

$$
\begin{gathered}
K_{\chi}(\theta):=\gamma_{1} \theta+L \chi \quad \forall \theta \in \mathbf{R}, \forall \chi \in[0,1] \\
G_{\chi}(w):=-\eta_{1}^{-1}(w) \chi+\eta_{0}^{-1}(w)(\chi-1) \quad \forall w \in[\tilde{\theta}, 0], \forall \chi \in[0,1]
\end{gathered}
$$

(see Figs. 4, 5), and $\chi$ fulfills (2.11). Here $u$ represents the enthalpy density. Later on $G_{\chi}(w)$ will be extended to the whole $\mathbf{R}$; however, just the values $\tilde{\theta} \leqslant w \leqslant 0$ are physically meaningful and then it will be quite convenient to prove maximum and minimum principles for $w$.

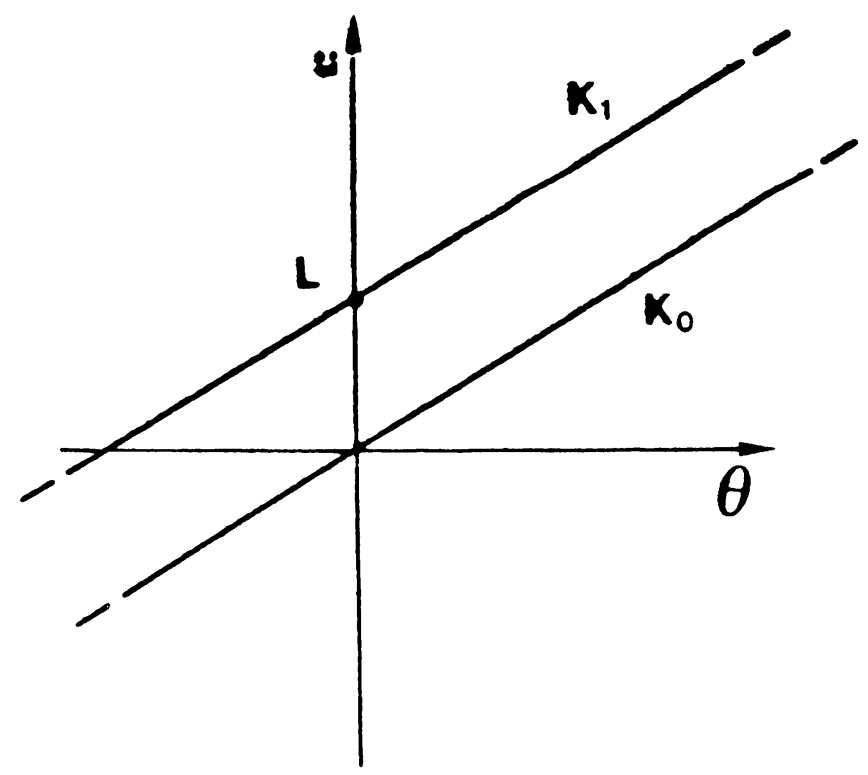

FIG. 4. Enthalpy density versus temperature for a binary alloy. $K_{0}$ : solid,$K_{1}$ : liquid. 


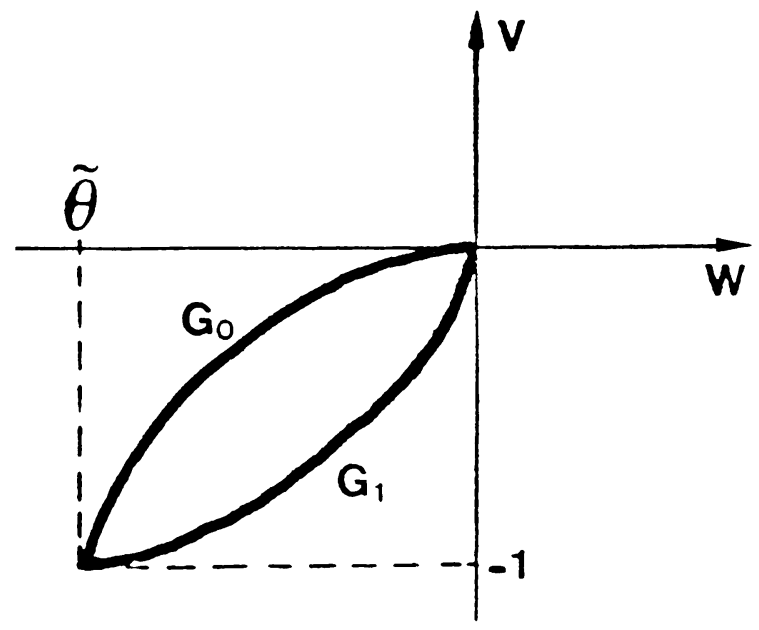

FIG. 5. Negative concentration versus chemical activity for a binary alloy. $G_{0}$ : solid, $G_{1}$ : liquid.

In [2] Bermudez and Saguez used (2.11) for eliminating $\chi$ from (2.18) and (2.19), getting

$$
\begin{array}{ll}
u \in \hat{K}_{w}(\theta):=K_{\tilde{H}\left(\theta-w^{\prime}\right)}(\theta) & \text { in } Q, \\
v \in \tilde{G}_{\theta}(w):=G_{\tilde{H}(\theta-w)}(w) & \text { in } Q ;
\end{array}
$$

then they used the maximal monotonicity of the graphs $\hat{K}_{w}(\cdot)$ and $\hat{G}_{\theta}(\cdot)$ (the latter extended for $w \notin[\hat{\theta}, 0])$, for fixed $\theta$ and $w$. Here we shall follow a different approach.

We notice that the system $(2.11),(2.14), \ldots,(2.19)$ can be directly deduced from physical principles, without any assumption on the regularity of the interface. In the framework of this weak formulation, it is possible to introduce heat and mass sources at the second members of (2.14) and (2.15), respectively. In such a case new phases can appear; in a more accurate physical model, nucleation phenomena should be considered.

If the solute concentration is "small", namely if $c$ is confined to a neighborhood of zero, it is possible to linearize the $\eta_{i}$ 's, setting

$$
\theta_{i}=\tilde{\eta}_{i}(c):=-\frac{1}{r_{i}} c \quad \text { on } \mathscr{S} \quad(i=0,1),
$$

where $r_{i}=-\left(\eta_{1}^{\prime}(0)\right)^{-1}$; thus $0<r_{0}<r_{1}<+\infty$ (see Fig. 6). Then we set $\gamma_{0}:=r_{0}, \ell:=r_{1}$ $-r_{0}$ and replace (2.19) with

$$
\tilde{G}_{\chi}(w):=\gamma_{0} w-\ell w^{-} \chi \quad \forall w \in \mathbf{R}, \forall \chi \in[0,1],
$$

(see Fig.7), where $w^{-}:=\frac{1}{2}(|w|-w)$.

If $\gamma_{1,1} \neq \gamma_{1,0}$, namely if the product between the specific heat and the density depends on the phase, then we replace (2.18) with

$$
\tilde{K}_{\chi}(\theta):=\gamma_{1,0} \theta+\left(\gamma_{1,1}-\gamma_{1,0}\right) \cdot(\theta+\zeta) \cdot \chi \quad \forall \theta \in \mathbf{R}, \forall \chi \in[0,1],
$$

where $\zeta \in \mathbf{R}$. This corresponds to a latent heat of the form

$$
L(\theta):=\left(\gamma_{1,1}-\gamma_{1,0}\right) \cdot(\theta+\zeta)
$$




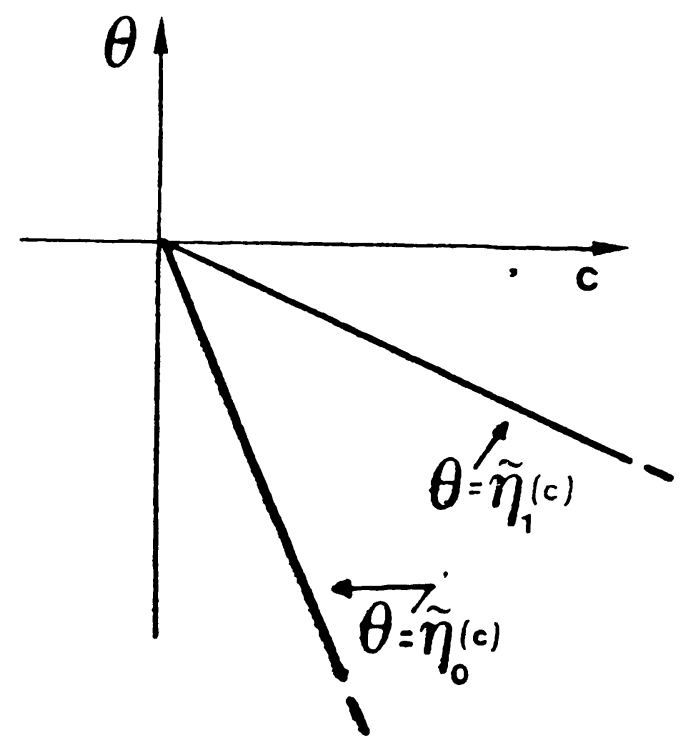

FIG. 6. Linearized phase diagram, for $c \cong 0$.

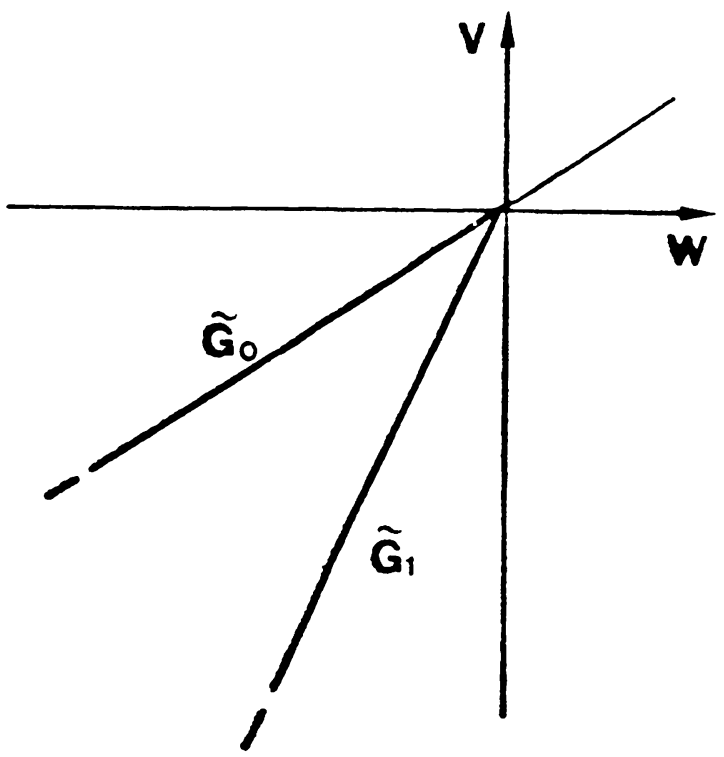

FIG. 7. Linearized concentration versus chemical activity relationship. 
$L(\theta)$ is negative either for large or for small values of $\theta$, depending on the sign of $\gamma_{1,1}-\gamma_{1,0}$; in order to prevent such a pathology, it is suitable to have either a maximum or a minimum principle at disposal; as we shall see, it is possible to deduce both.

Finally we assume that $\Omega$ is of class $C^{1}$ and introduce boundary and initial conditions:

$$
\begin{gathered}
\left.\overline{\bar{k}}_{1} \cdot \frac{\partial \theta}{\partial \nu}=p, \quad \overline{\bar{k}}_{2} \cdot \frac{\partial w}{\partial \nu}=q \quad \text { on } \partial \Omega \times\right] 0, T[, \\
\left.u\right|_{t=0}=u^{0},\left.\quad v\right|_{t=0}=v^{0} \quad \text { in } \Omega,
\end{gathered}
$$

where $p, q, u^{0}, v^{0}$ are given functions and $\partial / \partial \nu$ denotes the exterior normal derivative.

2. Now we remove the condition (2.6) of thermodynamic equilibrium and introduce supercooled and superheated states, corresponding to $\theta<w$ in the liquid and $\theta>w$ in the solid, respectively. We introduce the dynamical law

$$
\frac{\partial \chi}{\partial t}+\tilde{H}^{-1}(\chi) \ni \beta(\theta, w) \quad \text { in } Q,
$$

where $\beta \in C^{0}\left(\mathbf{R}^{2}\right)$ and $\operatorname{sign} \beta(\theta, w)=\operatorname{sign}(\theta-w)$, for any $\theta, w \in \mathbf{R}$; for instance

$$
\beta(\theta, w)=\mu_{1}(\theta-w)^{+}-\mu_{2}(\theta-w)^{-} \quad\left(\mu_{1}, \mu_{2} \text { : constants }>0\right) .
$$

(2.28) corresponds to the variational inequality (1.4) and can also be written in the form

$$
0 \leqslant \chi \leqslant 1 \quad \text { in } Q \text { and } \frac{\partial \chi}{\partial t}= \begin{cases}\beta(\theta, w)^{+} & \text {where } \chi=0 \\ \beta(\theta, w) & \text { where } 0<\chi<1 \\ -\beta(\theta, w)^{-} & \text {where } \chi=1\end{cases}
$$

(2.28) represents a relaxation toward the equilibrium condition (2.11).

3. Now we consider the case of a single dimension of space, with $\Omega=] 0, a[(a>0)$. We assume that the interface is of the form

$$
\mathscr{S}=\{(s(t), t) \mid 0 \leqslant t \leqslant T\},
$$

where $s \in C^{0}([0, T]), 0 \leqslant s(t) \leqslant a$, and that

$$
Q_{1}=\{(x, t) \mid 0<x<s(t), 0<t<T\}, \quad Q_{0}=\{(x, t) \mid s(t)<x<a, 0<t<T\} .
$$

Here $(2.1), \ldots,(2.4)$ can be rewritten in the form

$$
\begin{gathered}
\gamma_{1, i} \frac{\partial \theta}{\partial t}-\frac{\partial}{\partial x}\left(k_{1, i} \frac{\partial \theta}{\partial x}\right)=0 \quad \text { in } Q_{i}, \\
\frac{\partial c}{\partial t}-\frac{\partial}{\partial x}\left(\tilde{k}_{2, i} \frac{\partial c}{\partial x}\right)=0 \quad \text { in } Q_{i}, \\
\left.k_{1,0} \frac{\partial \theta}{\partial x}(s(t)+0, t)-k_{1,1} \frac{\partial \theta}{\partial x}(s(t)-0, t)=L s^{\prime}(t) \quad \text { in }\right] 0, T[, \\
\tilde{k}_{2,0} \frac{\partial c}{\partial x}(s(t)+0, t)-\tilde{k}_{2,1} \frac{\partial c}{\partial x}(s(t)-0, t) \\
\left.=\left[c_{0}(s(t), t)-c_{1}(s(t), t)\right] \cdot s^{\prime}(t) \quad \text { in }\right] 0, T[.
\end{gathered}
$$

Also, this setting corresponds to a system of the form $(2.11),(2.14), \ldots,(2.19)$. 
Here the phase characteristic function can be expressed in terms of $s$ :

$$
\chi(x, t) \in \tilde{H}(s(t)-x) \quad \text { in } Q,
$$

and the following kinetic law can be considered:

$$
\left.s^{\prime}(t)=\beta(\theta(s(t), t), w(s(t), t)), \quad \text { where } 0<s(t)<a, \text { in }\right] 0, T[
$$

with $\beta$ as above. We stress that even in a single space dimension, (2.28) and (2.36) are not equivalent.

Taking account of the constraint acting on $s(t),(2.36)$ will be coupled with

$$
s^{\prime}(t)= \begin{cases}\beta(\theta(0, t), w(0, t))^{+} & \text {where } s(t)=0 \\ -\beta(\theta(a, t), w(a, t))^{-} & \text {where } s(t)=a .\end{cases}
$$

(2.36) and (2.37) correspond to the following variational inequality:

$$
\left\{\begin{array}{l}
0 \leqslant s(t) \leqslant a ; \quad \forall \xi \in[0, a], \\
{\left[s^{\prime}(t)-\beta(\theta(s(t), t), w(s(t), t))\right] \cdot[\xi-s(t)] \geqslant 0 .}
\end{array}\right.
$$

(2.28) and (2.38) represent a relaxation toward the equilibrium conditions (2.11) and

$$
\beta(\theta(s(t), t), w(s(t), t)) \cdot[s(t)-\xi] \geqslant 0 \quad \forall \xi \in[0, a], \text { in }] 0, T[.
$$

3. One-dimensional case. We denote by $H$ the Heaviside function

$$
H(\xi):= \begin{cases}0 & \text { if } \xi \leqslant 0 \\ 1 & \text { if } \xi>0 .\end{cases}
$$

We also set $W:=L^{2}(0, a), V:=H^{1}(0, a)$, and

$$
v^{\prime}\left\langle A_{i} u, v\right\rangle_{V}:=k_{i} \int_{0}^{a} u^{\prime}(x) \cdot v^{\prime}(x) d x \quad \forall u, v \in V(i=1,2) .
$$

We assume that

$$
\begin{gathered}
\beta \in C^{0}\left(\mathbf{R}^{2}\right), \\
f_{i} \in L^{2}\left(0, T ; V^{\prime}\right) \quad(i=1,2) ; 0 \leqslant s^{0} \leqslant a ; \theta^{0}, \omega^{0} \in V^{\prime} .
\end{gathered}
$$

Problem (P1). Find $\theta, w \in L^{2}(0, T ; V)$ and $s \in W^{1,1}(0, T)$ such that

$$
\begin{gathered}
\left.\frac{\partial}{\partial t}\left[\gamma_{1} \theta+L H(s(t)-x)\right]+A_{1} \theta=f_{1} \quad \text { in } V^{\prime}, \text { a.e. in }\right] 0, T[ \\
\left.\frac{\partial}{\partial t}\left[\gamma_{0} w-\ell w^{-} H(s(t)-x)\right]+A_{2} w=f_{2} \quad \text { in } V^{\prime}, \text { a.e. in }\right] 0, T[, \\
\left\{\begin{array}{l}
\text { a.e. in }] 0, T[, \quad 0 \leqslant s(t) \leqslant a \text { and } \forall \xi \in[0, a] \\
{\left[s^{\prime}(t)-\beta(\theta(s(t), t), w(s(t), t)] \cdot[\xi-s(t)] \geqslant 0,\right.} \\
\left.\theta\right|_{t=0}=\theta^{0} \quad \text { in } V^{\prime}, \\
{\left[\gamma_{0} w-\ell w^{-} \cdot H(s(t)-x)\right]_{t=0}=\omega^{0} \quad \text { in } V^{\prime},} \\
s(0)=s^{0} .
\end{array}\right.
\end{gathered}
$$


Remarks. (i) (3.3) yields $\gamma_{1} \theta+L H(s(t)-x) \in H^{1}\left(0, T ; V^{\prime}\right)$; moreover $H(s(t)-x) \in$ $C^{0}([0, T] ; W)$; hence $\theta \in C^{0}\left([0, T] ; V^{\prime}\right)$ and this gives a meaning to (3.6). Similarly (3.4) yields $\gamma_{0} w-\ell w^{-} H(s(t)-x) \in H^{1}\left(0, T ; V^{\prime}\right)$ and this justifies (3.7). Notice that a priori we cannot give a meaning to $\left.w\right|_{t=0}$.

(ii) (3.3) can be rewritten in the following equivalent form:

$$
\begin{aligned}
\gamma_{1} \frac{d}{d t} \int_{0}^{a} \theta v d x+L s^{\prime}(t) \cdot v(s(t))+k_{1} \int_{0}^{a} \frac{\partial \theta}{\partial x} \cdot v^{\prime} d x & ={ }_{v^{\prime}}\left\langle f_{1}, v\right\rangle_{v} \\
\forall v & \in V, \text { a.e. in }] 0, T[
\end{aligned}
$$

(iii) For a suitable choice of $f_{1}$ and $f_{2}$, the variational problem (P1) is equivalent to the corresponding strong formulation, in which (3.3) and (3.4) are replaced by (2.31), ., (2.34). Hence the regularity properties of the solution can be improved.

Lemma 1. For any $\varepsilon>0$ there exists a constant $C(\varepsilon)>0$ such that, for any $\theta \in V$ and $r \in[0, a]$,

$$
|\theta(r)|^{2} \leqslant \int_{0}^{a}\left[\varepsilon \theta^{\prime}(\eta)^{2}+C(\varepsilon) \theta(\eta)^{2}\right] d \eta .
$$

Proof. Let $\xi \in[0, a]$ be such that $\theta(\xi)=\frac{1}{a} \int_{0}^{a} \theta(\eta) d \eta$. We have

$$
\begin{aligned}
\theta(r)^{2} & =\theta(\xi)^{2}+\int_{\xi}^{r}\left(\theta^{2}(\eta)\right)^{\prime} d \eta \\
& \leqslant\left[\frac{1}{a} \int_{0}^{a} \theta(\eta) d \eta\right]^{2}+2\left|\int_{\xi}^{r} \theta(\eta) \theta^{\prime}(\eta) d \eta\right| \\
& \leqslant \int_{0}^{a}\left[\frac{1}{a} \theta(\eta)^{2}+\varepsilon \theta^{\prime}(\eta)^{2}+\frac{1}{\varepsilon} \theta(\eta)^{2}\right] d \eta
\end{aligned}
$$

THEOREM 1. Assume that (3.1), (3.2) hold and that

$$
\begin{gathered}
|\beta(\xi, \eta)| \leqslant \text { Constant } \quad \forall \xi, \eta \in \mathbf{R}, \\
\theta^{0}, \omega^{0} \in W .
\end{gathered}
$$

Then problem (P1) has at least one solution such that

$$
\begin{gathered}
\theta \in L^{2}(0, T ; V) \cap L^{\infty}(0, T ; W) \cap H^{1}\left(0, T ; V^{\prime}\right), \\
w \in L^{2}(0, T ; V) \cap L^{\infty}(0, T ; W) \cap H^{1 / 2-\delta}(0, T ; W), \quad \forall \delta>0, \\
s \in W^{1, \infty}(0, T) .
\end{gathered}
$$

REMARK. The assumption (3.11) of uniform boundedness is quite restrictive; however, maximum and minimum principles can be proved and then (3.11) can be removed, as in Theorem 2 below.

Proof. (i) Faedo-Galerkin approximation

Let $\left\{V_{m}\right\}_{m \in \mathbf{N}}$ be a sequence of finite-dimensional subspaces filling up $V$. Let

$$
\begin{array}{rlrl}
\left\{f_{i m} \in C^{\infty}\left([0, T] ; V^{\prime}\right)\right\}_{m \in \mathbf{N}}, & f_{i m} & \rightarrow f_{i} \text { strongly in } L^{2}\left(0, T ; V^{\prime}\right)(i=1,2), \\
\left\{\theta_{m}^{0} \in V_{m}\right\}_{m \in \mathbf{N}}, & \theta_{m}^{0} \rightarrow \theta^{0} \text { strongly in } W, \\
\left\{w_{m}^{0} \in V_{m}\right\}_{m \in \mathbf{N}}, & w_{m}^{0} \rightarrow w^{0} \text { strongly in } W,
\end{array}
$$


where $w^{0}$ is such that $\gamma_{0} w^{0}-\ell\left(w^{0}\right)^{-} \cdot H\left(s^{0}-x\right)=\omega^{0}$ a.e. in $] 0, a[$,

$$
\begin{aligned}
& H_{m}(\xi):=\max \left\{0, \min \left[\frac{1}{2}(m \xi+1), 1\right]\right\} \quad \forall \xi \in \mathbf{R}, \\
& \Phi_{m}(\xi):=\frac{1}{m}\left[(\xi-a)^{+}-\xi^{-}\right] \quad \forall \xi \in \mathbf{R} .
\end{aligned}
$$

For any $m$ we introduce the following approximated problem.

Problem $(\mathrm{P} 1)_{m}$. Find $\theta_{m}, w_{m}:\left[0, T\left[\rightarrow V_{m}\right.\right.$ and $s_{m}:[0, T[\rightarrow \mathbf{R}$ such that, setting $\tilde{s}_{m}(t):=\max \left[\min \left(s_{m}(t), a\right), 0\right]$,

$$
\begin{gathered}
\int_{0}^{a}\left[\gamma_{1} \frac{\partial \theta_{m}}{\partial t} v+k_{1} \frac{\partial \theta_{m}}{\partial t} \cdot v^{\prime}\right] d x+L \tilde{s}_{m}^{\prime}(t) \cdot v\left(\tilde{s}_{m}(t)\right)={ }_{v^{\prime}}\left\langle f_{1 m}(t), v\right\rangle_{V} \\
\left.\forall v \in V_{m}, \text { in }\right] 0, T[ \\
\int_{0}^{a}\left\{\left[\gamma_{0}+\ell H_{m}\left(-w_{m}\right) \cdot H_{m}\left(\tilde{s}_{m}(t)-x\right)\right] \frac{\partial w_{m}}{\partial t} v+k_{2} \frac{\partial w_{m}}{\partial x} \cdot v^{\prime}\right\} d x \\
\left.-\ell w_{m}\left(\tilde{s}_{m}(t), t\right)^{-} \cdot \tilde{s}_{m}^{\prime}(t) \cdot v\left(\tilde{s}_{m}(t)\right)={ }_{V^{\prime}}\left\langle f_{2 m}(t), v\right\rangle_{V} \quad \forall v \in V_{m}, \text { in }\right] 0, T[ \\
\left.s_{m}^{\prime}(t)+\Phi_{m}\left(s_{m}(t)\right)=\beta\left(\theta_{m}\left(\tilde{s}_{m}(t), t\right), w_{m}\left(\tilde{s}_{m}(t), t\right)\right) \text { in }\right] 0, T[ \\
\left.\int_{0}^{a} \theta_{m} v d x\right|_{t=0}=\int_{0}^{a} \theta^{0} v d x \quad \forall v \in V_{m} \\
\left.\int_{0}^{a} w_{m} v d x\right|_{t=0}=\int_{0}^{a} w^{0} v d x \quad \forall v \in V_{m} \\
s_{m}(0)=s^{0} .
\end{gathered}
$$

$(\mathrm{P} 1)_{m}$ is equivalent to a Cauchy problem for a system of a finite number of ordinary differential equations and has at least one solution in $\left[0, T_{m}\left[\right.\right.$, for a suitable $\left.\left.T_{m} \in\right] 0, T\right]$.

(ii) A priori estimate

(3.19) and (3.11) yield

$$
\begin{aligned}
\left|\tilde{s}_{m}^{\prime}(t)\right| & =\left|\beta\left(\theta_{m}\left(\tilde{s}_{m}(t), t\right), w_{m}\left(\tilde{s}_{m}(t), t\right)\right)\right| \\
& \leqslant \text { Constant (independent of } m), \text { in }] 0, T[.
\end{aligned}
$$

Now we take $v=\theta_{m}$ in (3.17), $v=w_{m}$ in (3.18) and sum these equations. We notice that by (3.23) and by Lemma 1 we have

$$
\begin{aligned}
& \left|L \tilde{s}_{m}^{\prime}(t) \cdot \theta\left(\tilde{s}_{m}(t), t\right)-\ell w_{m}\left(\tilde{s}_{m}(t), t\right)^{-} \cdot \tilde{s}_{m}^{\prime}(t) \cdot w_{m}\left(\tilde{s}_{m}(t), t\right)\right| \\
& \leqslant \text { Constant } \cdot\left\{\left|\theta_{m}\left(\tilde{s}_{m}(t), t\right)\right|+\left[w_{m}\left(\tilde{s}_{m}(t), t\right)^{-}\right]^{2}\right\} \\
& \leqslant \text { Constant } \cdot\left(\varepsilon^{1 / 2}\left\|\theta_{m}\right\|_{V}+C(\varepsilon)^{1 / 2}\left\|\theta_{m}\right\|_{W}+\varepsilon\left\|w_{m}^{-}\right\|_{V}^{2}+C(\varepsilon)\left\|w_{m}^{-}\right\|_{W}^{2}\right),
\end{aligned}
$$


for any $\varepsilon>0$. Setting $B_{m}(\xi):=\int_{0}^{\xi} H_{m}(-\eta) \eta d \eta \forall \xi \in \mathbf{R}$ (thus $B_{m}(\xi) \rightarrow \frac{1}{2}\left(\xi^{-}\right)^{2}$ ), we have

$$
\begin{aligned}
& \int_{0}^{a} H_{m}\left(-w_{m}\right) \cdot H_{m}\left(\tilde{s}_{m}(t)-x\right) \cdot \frac{\partial w_{m}}{\partial t} \cdot w_{m} d x \\
&=\int_{0}^{a} H_{m}\left(\tilde{s}_{m}(t)-x\right) \cdot \frac{\partial}{\partial t} B_{m}\left(w_{m}\right) d x \\
&=\frac{d}{d t} \int_{0}^{a}\left[H_{m}\left(\tilde{s}_{m}(t)-x\right) \cdot B_{m}\left(w_{m}(x, t)\right)\right] \\
&-\int_{0}^{a} H_{m}^{\prime}\left(\tilde{s}_{m}(t)-x\right) \cdot \tilde{s}_{m}^{\prime}(t) \cdot B_{m}\left(w_{m}(x, t)\right) d x ;
\end{aligned}
$$

moreover, still by (3.23) and by Lemma 1, we have

$$
\begin{aligned}
& \ell\left|\int_{0}^{a} H_{m}^{\prime}\left(\tilde{s}_{m}(t)-x\right) \cdot \tilde{s}_{m}^{\prime}(t) \cdot B_{m}\left(w_{m}(x, t)\right) d x\right| \\
& \leqslant \text { Constant } \cdot B_{m}\left(w_{m}\left(\tilde{s}_{m}(t), t\right)\right)+\sigma(m) \\
& \leqslant \text { Constant } \cdot\left(\varepsilon\left\|w_{m}^{-}\right\|_{V}^{2}+C(\varepsilon)\left\|w_{m}^{-}\right\|_{W}^{2}\right)+\sigma(m),
\end{aligned}
$$

where $\sigma(m) \rightarrow 0$ as $m \rightarrow \infty$. Thus we get

$$
\begin{aligned}
\frac{\gamma_{1}}{2} \frac{d}{d t} & \int_{0}^{a} \theta_{m}(x, t)^{2} d x+k_{1} \int_{0}^{a}\left(\frac{\partial \theta_{m}}{\partial x}\right)^{2} d x+\frac{\gamma_{0}}{2} \frac{d}{d t} \int_{0}^{a} w_{m}(x, t)^{2} d x \\
& +k_{2} \int_{0}^{a}\left(\frac{\partial w_{m}}{\partial x}\right)^{2} d x+\ell \frac{d}{d t} \int_{0}^{a} H_{m}(s(t)-x) \cdot B_{m}\left(w_{m}(x, t)\right) d x \\
\leqslant & \left\|f_{1}\right\|_{V^{\prime}} \cdot\left\|\theta_{m}\right\|_{V}+\left\|f_{2}\right\|_{V^{\prime}} \cdot\left\|w_{m}\right\|_{V} \\
& + \text { Constant } \cdot\left(\varepsilon^{1 / 2}\left\|\theta_{m}\right\|_{V}+C(\varepsilon)^{1 / 2}\left\|\theta_{m}\right\|_{w}+\varepsilon\left\|w_{m}^{-}\right\|_{V}^{2}+C(\varepsilon)\left\|w_{m}^{-}\right\|_{w}^{2}\right)+\sigma(m) .
\end{aligned}
$$

Applying Gronwall's lemma we get $T_{m}=T$ for any $m$ and

$\left\|\theta_{m}\right\|_{L^{\infty}(0, T ; W) \cap L^{2}(0, T ; V)},\left\|w_{m}\right\|_{L^{\infty}(0, T ; W) \cap L^{2}(0, T ; V)} \leqslant$ Constant (independent of $m$ );

hence we have

$$
\left\|\theta_{m}\left(s_{m}(t), t\right)\right\|_{L^{2}(0, T)},\left\|w_{m}\left(s_{m}(t), t\right)\right\|_{L^{2}(0, T)} \leqslant \text { Constant }
$$

and by comparison in (3.17) we also get

$$
\left\|\theta_{m}\right\|_{H^{1}\left(0, T ; V^{\prime}\right)} \leqslant \text { Constant. }
$$

Now we want to estimate the time regularity of $w_{m}$. We first notice that by comparison in (3.18) and by (3.25) we have

$$
\left\|\left[\gamma_{0}+\ell H_{m}\left(-w_{m}\right) \cdot H_{m}\left(s_{m}(t)-x\right)\right] \cdot \frac{\partial w_{m}}{\partial t}\right\|_{I^{2}\left(0, T ; V^{\prime}\right)} \leqslant \text { Constant. }
$$

For any $m, w_{m}$ is absolutely continuous in time, by (3.18); hence, setting $w_{m}^{h}(x, t):=w_{m}(x, t+h)$ in $Q$ (here $\left.w_{m}(x, T+\delta):=w_{m}(x, T) \forall \delta>0\right)$, as $h \rightarrow 0^{+}$we have

$$
\sigma_{h}^{m}(x, t):=\frac{\partial w_{m}}{\partial t}-\frac{w_{m}^{h}-w_{m}}{h} \rightarrow 0 \text { strongly in } L^{2}\left(0, T ; V^{\prime}\right)
$$


Then we get

$$
\begin{gathered}
\gamma_{0} \iint_{Q} \frac{\left(w_{m}^{h}-w_{m}\right)^{2}}{h} d x d t \leqslant \iint_{Q}\left[\gamma_{0}+\ell H_{m}\left(-w_{m}\right) \cdot H_{m}\left(s_{m}(t)-x\right)\right] \cdot \frac{\left(w_{m}^{h}-w_{m}\right)^{2}}{h} d x d t \\
\quad=\iint_{Q}\left[\gamma_{0}+\ell H_{m}\left(-w_{m}\right) \cdot H_{m}\left(s_{m}(t)-x\right)\right] \cdot\left(\frac{\partial w_{m}}{\partial t}+\sigma_{m h}\right) \cdot\left(w_{m}^{h}-w_{m}\right) d x d t \\
\leqslant \text { Constant } \cdot\left\|w_{m}^{h}-w_{m}\right\|_{L^{2}(0, T: V)}+\hat{\sigma}_{m h} \leqslant \text { Constant }
\end{gathered}
$$

(here $\hat{\sigma}_{m h} \rightarrow 0$ as $h \rightarrow 0^{+}$). For any $\left.\left.\delta \in\right] 0, \frac{1}{2}\right]$ and any $u \in H^{1 / 2-\delta}(\mathbf{R})$, one has (see [22], p. 190)

$$
\begin{aligned}
\|u\|_{H^{1 / 2-\delta}(\mathbf{R})} & =\|u\|_{L^{2}(\mathbf{R})}+\left\{\iint_{\mathbf{R}^{2}} \frac{[u(t)-u(\tau)]^{2}}{|t-\tau|^{2-2 \delta}} d t d \tau\right\}^{1 / 2} \\
& =\|u\|_{L^{2}(\mathbf{R})}+\left\{2 \int_{R^{+}} d h h^{2 \delta-1} \cdot \int_{\mathbf{R}} \frac{[u(t+h)-u(t)]^{2}}{h} d t\right\}^{1 / 2} .
\end{aligned}
$$

Thus we get

$$
\left\|w_{m}\right\|_{H^{1 / 2-\delta}(0, T: W)} \leqslant \text { Constant }, \quad \forall \delta>0 .
$$

We notice that a similar estimate holds also for $\theta_{m}$, as a consequence of (3.24) and (3.26).

(iii) Limit procedure

By the previous a priori estimates, there exist $\theta, w, s$ such that, possibly taking subsequences,

$$
\begin{gathered}
\theta_{m} \rightarrow \theta, \quad w_{m} \rightarrow w \\
\text { weakly star in } L^{\infty}(0, T ; W) \cap H^{1 / 2-\delta}(0, T ; W) \cap L^{2}(0, T ; V) \quad \forall \delta>0 \\
s_{m}, \tilde{s}_{m} \rightarrow s \text { weakly star in } W^{1, \infty}(0, T) .
\end{gathered}
$$

By Aubin's lemma (cf. [17, p. 57]), (3.30) yields

$$
\theta_{m} \rightarrow \theta, \quad w_{m} \rightarrow w \quad \text { strongly in } L^{2}\left(0, T ; H^{1-\rho}(0, a)\right), \quad \forall \rho>0 ;
$$

then for any $\rho \in] \frac{1}{2}, 1[$, we have

$$
\begin{aligned}
\| \theta_{m}\left(\tilde{s}_{m}(t)\right. & , t)-\theta(s(t), t) \|_{L^{2}(0, T)} \\
& \leqslant\left\|\theta_{m}\left(\tilde{s}_{m}(t), t\right)-\theta_{m}(s(t), t)\right\|_{L^{2}(0, T)}+\left\|\theta_{m}(s(t), t)-\theta(s(t), t)\right\|_{L^{2}(0, T)} \\
& \leqslant\left\|\int_{s(t)}^{\tilde{s}_{m}(t)} \frac{\partial \theta_{m}}{\partial x}(\xi, t) d \xi\right\|_{L^{2}(0, T)}+\text { Constant } \cdot\left\|\theta_{m}-\theta\right\|_{L^{2}\left(0, T ; H^{1 / 2+\rho}(0, a)\right)} \rightarrow 0
\end{aligned}
$$

that is,

$$
\theta_{m}\left(\tilde{s}_{m}(t), t\right) \rightarrow \theta(s(t), t) \quad \text { strongly in } L^{2}(0, T)
$$

Similarly we get

$$
w_{m}\left(\tilde{s}_{m}(t), t\right) \rightarrow w(s(t), t) \quad \text { strongly in } L^{2}(0, T)
$$

and then

$$
\beta\left(\theta_{m}\left(\tilde{s}_{m}(t), t\right), w_{m}\left(\tilde{s}_{m}(t), t\right)\right) \rightarrow \beta(\theta(s(t), t), w(s(t), t))
$$

weakly star in $L^{\infty}(0, T)$ and strongly in $L^{2}(0, T)$. 
Now we set $b_{m}(\xi):=\int_{0}^{\xi} H_{m}(-\eta) d \eta, \forall \xi \in \mathbf{R}$. For any $v \in V$ we have

$$
\begin{aligned}
\int_{0}^{a} H_{m}\left(-w_{m}\right) \cdot H_{m}\left(\tilde{s}_{m}(t)-x\right) \frac{\partial w_{m}}{\partial t} v d x=\int_{0}^{a} H_{m}\left(\tilde{s}_{m}(t)-x\right) \frac{\partial b_{m}\left(w_{m}\right)}{\partial t} v d x \\
\quad=\frac{d}{d t} \int_{0}^{a} H_{m}\left(\tilde{s}_{m}(t)-x\right) \cdot b_{m}\left(w_{m}\right) v d x-\int_{0}^{a} H_{m}^{\prime}\left(\tilde{s}_{m}(t)-x\right) \cdot \tilde{s}_{m}^{\prime}(t) \cdot b_{m}\left(w_{m}\right) v d x ;
\end{aligned}
$$

we notice that by (3.32)

$$
b_{m}\left(w_{m}\right) \rightarrow-w^{-} \text {strongly in } L^{2}\left(0, T ; H^{1-\rho}(0, a)\right) \quad \forall \rho>0 .
$$

Thus taking $m \rightarrow \infty$ in (3.17) and (3.18) we get (3.3) and (3.4). Multiplying (3.19) by $\xi-s_{m}(t)$ for a generic $\xi \in[0, a]$, we have

$$
\begin{aligned}
{\left[s_{m}^{\prime}(t)-\beta\left(\theta_{m}\left(\tilde{s}_{m}(t), t\right), w_{m}\left(\tilde{s}_{m}(t), t\right)\right)\right] \cdot\left[\xi-s_{m}(t)\right] } & =-\Phi_{m}\left(s_{m}(t)\right) \cdot\left[\xi-s_{m}(t)\right] \geqslant 0,
\end{aligned}
$$

and taking $m \rightarrow \infty$ we get (3.5).

Proposition 1 (Maximum principle for $\theta$ ). Let $M \in \mathbf{R}$. Assume that (3.1), (3.2) hold and that

$$
\begin{gathered}
\beta(\xi, \eta) \geqslant 0 \quad \forall \xi \geqslant M, \quad \forall \eta \in \mathbf{R}, \\
\left.\theta^{0} \in W, \quad \theta^{0}(x) \leqslant M \quad \text { a.e. in }\right] 0, a[, \\
f_{1} \leqslant 0 \quad \text { in } \mathscr{D}^{\prime}(Q) .
\end{gathered}
$$

Then for any solution of problem (P1)

$$
\theta \leqslant M \text { a.e. in } Q \text {. }
$$

Proof. We take $v=(\theta-M)^{+}$in (3.3) and integrate in time.

Proposition 2 (Maximum principle for $w$ ). Let $N \in \mathbf{R}$. Assume that (3.1), (3.2) hold and that

$$
\begin{aligned}
& \beta(\xi, \eta) \leqslant 0 \quad \forall \xi \in \mathbf{R}, \quad \forall \eta \in\left[-N^{-}, 0\right] \\
& \left\{\begin{array}{l}
\text { there exist } s^{0} \in[0, a] \text { and } w^{0} \in W \text { such that } \\
\left.\omega^{0}(x)=\gamma_{0} w^{0}(x)-\ell w^{0}(x) \cdot H\left(s^{0}-x\right) \quad \text { a.e. in }\right] 0, a[, \\
\left.w^{0}(x) \leqslant N \text { a.e. in }\right] 0, a[
\end{array}\right. \\
& f_{2} \leqslant 0 \text { in } \mathscr{D}^{\prime}(Q) .
\end{aligned}
$$

Then for any solution of problem (P1)

$$
w \leqslant N \text { a.e. in } Q \text {. }
$$

Proof. We multiply (3.4) by $(w-N)^{+}$and integrate in time.

Minimum principles can be similarly stated for $\theta$ and $w$. These results can be coupled in several ways; they also enable us to prove another existence theorem, where $\beta$ is not required to be bounded: 
Theorem 2. Let $M_{1}, M_{2}, N_{1}, N_{2} \in \mathbf{R}, M_{1}<M_{2}$ and $N_{1}<N_{2}$. Assume that (3.1) holds and that

$$
\begin{gathered}
\theta^{0} \in W, \quad M_{1} \leqslant \theta^{0}(x) \leqslant M_{2} \text { a.e. in } \Omega \\
\left\{\begin{array}{l}
\text { there exist } w^{0} \in W \text { and } s^{0} \in[0, a] \text { such that } \\
\left.\omega^{0}(x)=\gamma_{0} w^{0}(x)-\ell w^{0}(x)^{-} H\left(s^{0}-x\right) \quad \text { a.e. in }\right] 0, a[ \\
\left.N_{1} \leqslant w^{0}(x) \leqslant N_{2} \quad \text { a.e. in }\right] 0, a[
\end{array}\right. \\
\left.\left.\beta(\xi, \eta) \leqslant 0 \quad \forall(\xi, \eta) \in(]-\infty, M_{1}\right] \times\left[N_{1}, N_{2}\right]\right) \cup\left(\left[M_{1}, M_{2}\right] \times\left[N_{2},+\infty[),\left(3, N_{1}, N_{2}\right] \cap \mathbf{R}^{-}\right)\right\} \\
\beta(\xi, \eta) \geqslant 0 \quad \forall(\xi, \eta) \in\left\{\left[M_{2},+\infty\left[\times\left(\left[M_{1}, M_{2}\right] \times\right]-\infty, N_{1}\right] \cap \mathbf{R}^{-}\right)\right. \\
f_{1}=f_{2}=0 \quad \text { in } \mathscr{D}^{\prime}(Q)
\end{gathered}
$$

Then problem (P1) has at least one solution such that

$$
M_{1} \leqslant \theta \leqslant M_{2}, \quad N_{1} \leqslant w \leqslant N_{2} \text { a.e. in } Q .
$$

Proof. We truncate $\beta$ outside $\left[M_{1}, M_{2}\right] \times\left[N_{1}, N_{2}\right]$, denote this new function by $\tilde{\beta}$ and replace $\beta$ with $\tilde{\beta}$ in (3.5). The problem we get has at least one solution by Theorem 1 . The maximum and minimum principles for $\theta$ yield $M_{1} \leqslant \theta \leqslant M_{2}$ a.e. in $Q$; then the maximum and minimum principles for $w$ yield $N_{1} \leqslant w \leqslant N_{2}$ a.e. in $Q$. Hence $\tilde{\beta}(\theta, w)=$ $\beta(\theta, w)$ and consequently $(\theta, w)$ solves also (P1).

Remark. The uniqueness of the solution of (P1) is an open question.

Generalizations. (i) Theorems 1 and 2 are easily extended to the case of nonconstant and phase-dependent diffusion coefficients, namely for $k_{i}=\hat{k}_{i}(\theta, w, H(s(t)-x))(i=$ $1,2)$, if the given functions $\hat{k}_{1}$ and $\hat{k}_{2}$ are continuous and comprised between two positive constants.

(ii) The previous results can be generalized to the case of $\gamma_{1}=\hat{\gamma}_{1}(\theta), \gamma_{0}=\hat{\gamma}_{0}(w)$, and $\ell=\hat{\ell}(w)$, under natural assumptions for the given functions $\hat{\gamma}_{1}, \hat{\gamma}_{0}$, and $\hat{\ell}$. Notice that this includes the constitutive relationship considered in Section 2 and corresponding to Figs. 3 and 5.

(iii) The results of this section can be extended to the case of phase-dependent specific heat and of temperature-dependent latent heat: $d e=\hat{\gamma}_{1}(\chi) d \theta+\hat{L}(\theta) d \chi(e=$ enthalpy density), with $\hat{\gamma}_{1}^{\prime}=\hat{L}^{\prime}$. Then the structure of (3.3) becomes similar to that of (3.4) and the regularity property $\theta \in H^{1}\left(0, T ; V^{\prime}\right)$ must be replaced by $\theta \in H^{1 / 2-\delta}(0, T ; W)$ for any $\delta>0$, which is deduced as in (3.27), ., (3.29).

(iv) Now we study the asymptotic behavior as the mass diffusivity $k_{2}$ vanishes in the solid phase. First, for any $s \in[0, a]$ we set

$$
\begin{gathered}
v^{\prime}\left\langle A_{2, \varepsilon}^{s} u, v\right\rangle_{V}:=k_{2} \int_{0}^{s} u^{\prime} v d x+\varepsilon \int_{0}^{a} u^{\prime} v^{\prime} d x \quad \forall u, v \in V, \forall \varepsilon>0, \\
v^{\prime}\left\langle A_{2}^{s} u, v\right\rangle_{V}:=k_{2} \int_{0}^{s} u^{\prime} v^{\prime} d x \quad \forall u \in H^{1}(0, s), \forall v \in V .
\end{gathered}
$$

Problem $(\mathrm{P} 1)_{\varepsilon}$. As (P1), with $A_{2}$ replaced by $A_{2, \varepsilon}^{s(t)}$ a.e. in $] 0, T[$. 
Theorem 3. Assume that (3.1), (3.2), (3.11), and (3.12) hold. For any $\varepsilon>0$ let $\left(\theta_{\varepsilon}, w_{\varepsilon}, s_{\varepsilon}\right)$ be a solution of problem $(\mathrm{P} 1)_{\varepsilon}$ (existing by Theorem 1$)$. Then there exist $\theta, w$, and $s$ such that, possibly taking subsequences,

$$
\begin{array}{ll}
\theta_{\varepsilon} \rightarrow \theta \quad \text { weakly star in } L^{\infty}(0, T ; W) \cap L^{2}(0, T ; V) \cap H^{1}\left(0, T ; V^{\prime}\right), \\
w_{\varepsilon} \rightarrow w \quad \text { weakly star in } L^{\infty}(0, T ; W) \cap H^{1 / 2-\delta}(0, T ; W), \quad \forall \delta>0, \\
s_{\varepsilon} \rightarrow s \quad \text { weakly star in } W^{1, \infty}(0, T) .
\end{array}
$$

Moreover, $(\theta, w, s)$ solves the reduced problem $(\mathrm{P} 1)_{0}$.

Problem $(\mathrm{P} 1)_{0}$. Find $\theta \in L^{2}(0, T ; V), w \in L^{2}(0, T ; W)$, and $s \in W^{1.1}(0, T)$ such that $\partial w / \partial x \in L^{2}(\{(x, t) \in Q \mid x<s(t)$ in $] 0, T[\})$ and

$$
\begin{aligned}
& \left.\frac{\partial}{\partial t}\left[\gamma_{1} \theta+L H(s(t)-x)\right]+A_{1} \theta=f_{1} \quad \text { in } V^{\prime} \text {, a.e. in }\right] 0, T[, \\
& \left.\frac{\partial}{\partial t}\left[\gamma_{0} w-\ell w^{-} H(s(t)-x)\right]+A_{2}^{s(t)} w=f_{2} \quad \text { in } V^{\prime} \text {, a.e. in }\right] 0, T[\text {, } \\
& \left\{\begin{array}{l}
\text { a.e. in }] 0, T[, \quad 0 \leqslant s(t) \leqslant a \text { and } \forall \xi \in[0, a] \\
{\left[s^{\prime}(t)-\beta\left(\theta(s(t), t), \lim _{x \rightarrow s(t)} w(x, t)\right)\right] \cdot[\xi-s(t)] \geqslant 0,}
\end{array}\right. \\
& \left.\theta\right|_{t=0}=\theta^{0} \text { in } V^{\prime} \text {, } \\
& {\left[\gamma_{0} w-\ell w^{-} H(s(t)-x)\right]_{t=0}=\omega^{0} \quad \text { in } V^{\prime} \text {, }} \\
& s(0)=s^{0} \text {. }
\end{aligned}
$$

Proof. Also here we have an estimate of the form of (3.23). We multiply (3.3) by $\theta_{\varepsilon}$ and (3.4) $)_{\varepsilon}$ by $w_{\varepsilon}$; by the procedure used in the proof of Theorem 1, we get

$$
\left\{\begin{array}{l}
\left\|\theta_{\varepsilon}\right\|_{L^{\infty}(0, T: W) \cap L^{2}(0, T: V)}, \quad\left\|w_{\varepsilon}\right\|_{L^{\infty}(0, T: W),} \\
\left.\left\|\left[k_{2} H\left(s_{\varepsilon}(t)-x\right)+\sqrt{\varepsilon}\right] \frac{\partial w_{\varepsilon}}{\partial x}\right\|_{L^{2}(Q)} \leqslant \text { Constant (independent of } \varepsilon\right),
\end{array}\right.
$$

whence estimates of the form of (3.26), (3.27), and (3.29), uniform in $\varepsilon$.

By these estimates, there exist $\theta, w, s, \xi$ such that, possibly taking subsequences, (3.48), .., (3.50) hold and

$$
H\left(s_{\varepsilon}(t)-x\right) \frac{\partial w_{\varepsilon}}{\partial x} \rightarrow \xi \quad \text { weakly in } L^{2}(Q)
$$

by the uniform convergence of $s_{\varepsilon}$ to $s$, we get

$$
\left.\xi=H(s(t)-x) \frac{\partial w}{\partial x} \quad \text { a.e. in }\right] 0, T[
$$

moreover, by (3.57) we have

$$
\varepsilon\left[1-H\left(s_{\varepsilon}(t)-x\right)\right] \frac{\partial w_{\varepsilon}}{\partial x} \rightarrow 0 \text { strongly in } L^{2}(Q) .
$$

By (3.49) and (3.50), we have that, possibly extracting a further subsequence, for any $n \in \mathbf{N}$

$$
\left.w_{\varepsilon} \rightarrow w \quad \text { uniformly in } C^{0}(] 0, s(t)-\frac{1}{n}[), \quad \text { a.e. in }\right] 0, T[\text {; }
$$


hence, still taking advantage of the uniform convergence of $s_{\varepsilon}$ to $s$,

$$
\left.\lim _{\varepsilon \rightarrow 0} w_{\varepsilon}\left(s_{\varepsilon}(t), t\right)=\lim _{x \rightarrow(t)^{-}} w(x, t), \quad \text { a.e. in }\right] 0, T[.
$$

Thus taking $\varepsilon \rightarrow 0$ in $(\mathrm{P} 1)_{\varepsilon}$ we get $(\mathrm{P} 1)_{0}$.

Remark. The interpretation of (3.52) is easily obtained by setting $\hat{k}_{2.0}=0$ in (2.32) and (2.34). We notice that here $w$ is no longer continuous across $\mathscr{S}$.

4. Case of several space dimensions. We remind the reader that we denote by $\tilde{H}$ the Heaviside graph; we also set $W:=L^{2}(\Omega), V:=H^{1}(\Omega)$ and

$$
{ }^{\prime}\left\langle A_{i} u, v\right\rangle_{v}:=\int_{\Omega} \overline{\bar{k}}_{i} \bar{\nabla} u \cdot \bar{\nabla} v d x \quad \forall u, v \in V(i=1,2) .
$$

We assume that

$$
\begin{gathered}
\beta \in C^{0}\left(\mathbf{R}^{2}\right), \\
\left\{\begin{array}{l}
\theta^{0}, \omega^{0} \in V^{\prime}, \quad f_{i} \in L^{2}\left(0, T ; V^{\prime}\right) \quad(i=1,2), \\
\chi^{0}: \Omega \rightarrow[0,1] \text { measurable. }
\end{array}\right.
\end{gathered}
$$

Problem (P2). Find $\theta, w \in L^{2}(0, T ; V)$ and $\chi \in H^{1}(0, T ; W)$ such that

$$
\begin{gathered}
\left.\frac{\partial}{\partial t}\left(\gamma_{1} \theta+L \chi\right)+A_{1} \theta=f_{1} \quad \text { in } V^{\prime}, \text { a.e. in }\right] 0, T[, \\
\left.\frac{\partial}{\partial t}\left(\gamma_{0} w-\ell w^{-} \chi\right)+A_{2} w=f_{2} \quad \text { in } V^{\prime}, \text { a.e. in }\right] 0, T[, \\
\frac{\partial \chi}{\partial t}+\tilde{H}^{-1}(\chi) \ni \beta(\theta, w) \quad \text { a.e. in } Q, \\
\left.\theta\right|_{t=0}=\theta^{0} \quad \text { in } V^{\prime}, \\
{\left[\gamma_{0} w-\ell w^{-} \chi\right]_{t=0}=\omega^{0} \quad \text { in } V^{\prime},} \\
\left.\chi\right|_{t=0}=\chi^{0} \quad \text { a.e. in } \Omega .
\end{gathered}
$$

Remark. (4.3) and (4.4) yield $\gamma_{1} \theta+L \chi, \gamma_{0} w-\ell w^{-} \chi \in H^{1}\left(0, T ; V^{\prime}\right)$; hence $\theta \in$ $H^{1}\left(0, T ; V^{\prime}\right)$ and (4.6), (4.7) are meaningful. Notice that $\left.w\right|_{t=0}$ is not meaningful, a priori.

THEOREM 4. Assume that (4.1), (4.2) hold and that

$$
\begin{gathered}
|\beta(\xi, \eta)| \leqslant \text { Constant }, \forall \xi, \eta \in \mathbf{R}, \\
\boldsymbol{\theta}^{0}, \omega^{0} \in W .
\end{gathered}
$$

Then problem (P2) has at least one solution such that

$$
\begin{aligned}
& \theta \in L^{\infty}(0, T ; W) \cap L^{2}(0, T ; V) \cap H^{1}\left(0, T ; V^{\prime}\right), \\
& w \in L^{\infty}(0, T ; W) \cap L^{2}(0, T ; V) \cap H^{1 / 2-\delta}(0, T ; W), \quad \forall \delta>0, \\
& \chi \in W^{1, \infty}\left(0, T ; L^{\infty}(\Omega)\right) .
\end{aligned}
$$

Remark. The boundedness assumption (4.9) can be removed by means of maximum and minimum principles, as in Section 3. 
Proof. (i) Faedo-Galerkin approximation

We introduce sequences $V_{m}, f_{i m}$, and $H_{m}$ as in the proof of Theorem 1 and such that

$$
\begin{gathered}
\left\{\chi_{m}^{0} \in V_{m}\right\}, \quad \chi_{m}^{0} \rightarrow \chi^{0} \quad \text { strongly in } W, \\
H_{m}^{n}(\xi):=H_{m}^{n}(\xi)-H_{m}^{n}(n-\xi) \quad \forall \xi \in \mathbf{R}, \\
b_{m}^{n}(\gamma x):=\int_{0}^{\xi} H_{m}^{n}(-\eta) d \eta, b^{n}(\xi):=\int_{0}^{\xi}[H(\eta)-H(n-\eta)] d \eta \quad \forall \xi \in \mathbf{R}, \\
\Psi_{m}(\xi):=m\left[(\xi-1)^{+}-\xi^{-}\right], \quad \forall \xi \in \mathbf{R} .
\end{gathered}
$$

For any $m, n \in \mathbf{N}$ we introduce the following approximated problem:

Problem $(\mathrm{P} 2)_{m}^{n}$. Find $\theta_{m}^{n}, w_{m}^{n}, \chi_{m}^{n}:\left[0, T\left[\rightarrow V_{m}\right.\right.$ such that

$$
\begin{gathered}
\left.\int_{\Omega}\left[\frac{\partial}{\partial t}\left(\gamma_{1} \theta_{m}^{n}+L \chi_{m}^{n}\right) \cdot v+\overline{\bar{k}}_{1} \cdot \bar{\nabla} \theta_{m}^{n} \cdot \bar{\nabla} v\right] d x={ }_{V^{\prime}}\left\langle f_{l m}, v\right\rangle_{V} \quad \forall v \in V_{m}, \text { in }\right] 0, T[ \\
\int_{\Omega}\left[\frac{\partial}{\partial t}\left(\gamma_{0} w_{m}^{n}+\ell b_{m}^{n}\left(w_{m}^{n}\right) \chi_{m}^{n}\right) \cdot v+\overline{\bar{k}}_{2} \cdot \bar{\nabla} w_{m}^{n} \cdot \bar{\nabla} v\right] d x={ }_{v^{\prime}}\left\langle f_{2 m}, v\right\rangle_{V} \\
\left.\quad \forall v \in V_{m}, \text { in }\right] 0, T[ \\
\left.\int_{\Omega}\left[\frac{\partial \chi_{m}^{n}}{\partial t}+\Psi_{m}\left(\chi_{m}^{n}\right)\right] v d x=\int_{\Omega} \beta\left(\theta_{m}^{n}, w_{m}^{n}\right) v d x \quad \forall v \in V_{m}, \text { in }\right] 0, T[ \\
\left.\int_{\Omega} \theta_{m}^{n} v d x\right|_{t=0}=\int_{\Omega} \theta_{m}^{0} v d x \quad \forall v \in V_{m} \\
\left.\int_{\Omega} w_{m}^{n} v d x\right|_{t=0}=\int_{\Omega} w_{m}^{0} v d x \quad \forall v \in V_{m} \\
\left.\int_{\Omega} \chi_{m}^{n} v d x\right|_{t=0}=\int_{\Omega} \chi_{m}^{0} v d x \quad \forall v \in V_{m}
\end{gathered}
$$

$(\mathrm{P} 2)_{m}^{n}$ is equivalent to a Cauchy problem for a system of ordinary differential equations and has at least one solution in $\left[0, T_{m}^{n}\left[\right.\right.$, for a suitable $\left.T_{m}^{n} \in\right] 0, T[$.

(ii) A priori estimates

We take $v=\theta_{m}^{n}$ in (4.14), $v=w_{m}^{n}$ in (4.15), $v=\partial \chi_{m}^{n} / \partial t$ in (4.16) and sum these equations. We set

$$
B_{m}^{n}(\chi):=\int_{0}^{\xi} H_{m}^{n}(-\eta) \eta d \eta \quad \forall \xi \in \mathbf{R} ;
$$

hence $\left|B_{m}^{n}(\xi)\right| \leqslant n^{2} / 2$. We notice that

$$
\begin{aligned}
\int_{\Omega} \frac{\partial}{\partial t}\left[b_{m}^{n}\left(w_{m}^{n}\right) \cdot \chi_{m}^{n}\right] \cdot w_{m}^{n} d x=\int_{\Omega}\left[\frac{\partial w_{m}^{n}}{\partial t} \cdot H_{m}^{n}\left(-w_{m}^{n}\right) \chi_{m}^{n}+b_{m}^{n}\left(w_{m}^{n}\right) \cdot \frac{\partial \chi_{m}^{n}}{\partial t}\right] w_{m} d x \\
\quad=\frac{d}{d t} \int_{\Omega} \chi_{m}^{n} \cdot B_{m}^{n}\left(w_{m}^{n}\right) d x-\int_{\Omega} \frac{\partial \chi_{m}^{n}}{\partial t} \cdot B_{m}^{n}\left(w_{m}^{n}\right) d x+\int_{\Omega} b_{m}^{n}\left(w_{m}^{n}\right) \cdot \frac{\partial \chi_{m}^{n}}{\partial t} \cdot w_{m}^{n} d x \\
\geqslant \frac{d}{d t} \int_{\Omega} \chi_{m}^{n} \cdot B_{m}^{n}\left(w_{m}^{n}\right) d x-\frac{n^{2}}{2} \int_{\Omega}\left|\frac{\partial \chi_{m}^{n}}{\partial t}\right| d x-n\left\|\frac{\partial \chi_{m}^{n}}{\partial t}\right\|_{W} \cdot\left\|w_{m}^{n}\right\|_{W}
\end{aligned}
$$


notice the presence of the factors $n$ and $n^{2}$. By a standard procedure based on the Gronwall's lemma, we get that $T_{m}^{n}=T^{n}$ for any $m$ and

$$
\left\{\begin{array}{l}
\left\|\theta_{m}^{n}\right\|_{L^{\infty}\left(0, T^{n}: W\right) \cap L^{2}\left(0, T^{n}: V\right)},\left\|w_{m}^{n}\right\|_{L^{\infty}\left(0, T^{n} ; W\right) \cap L^{2}\left(0, T^{n}: V\right)},\left\|\chi_{m}\right\|_{H^{1}\left(0, T^{n}: W\right)} \\
\leqslant \text { Constant }_{n}(: \text { constant dependent on } n \text { but not on } m)
\end{array}\right.
$$

then by comparing in (4.14) and by applying to (4.15) a technique similar to that used in Sec. 3, we also get

$$
\begin{aligned}
\left\|\theta_{m}^{n}\right\|_{H^{1}\left(0 . T^{n} \cdot V^{\prime}\right)} & \leqslant \text { Constant }_{n}, \\
\left\|w_{m}^{n}\right\|_{H^{1 / 2-\delta}\left(0, T^{n}: W\right)} & \leqslant \text { Constant }_{n}, \quad \forall \delta>0 .
\end{aligned}
$$

Setting $\left.Q_{n}:=\Omega \times\right] 0, T^{n}[$, by (4.9) we have

$$
\left\|\beta\left(\theta_{m}^{n}, w_{m}^{n}\right)\right\|_{L^{\infty}\left(Q_{n}\right)} \leqslant \text { Constant (independent of } n \text { and } m \text { ). }
$$

(iii) Limit as $m \rightarrow \infty$.

By the previous a priori estimates, for any $n \in \mathbf{N}$, there exist $\theta^{n}, w^{n}, \chi^{n}$ such that, possibly taking subsequences, as $m \rightarrow \infty$

$$
\begin{gathered}
\theta_{m}^{n} \rightarrow \theta^{n} \quad \text { weakly star in } L^{\infty}\left(0, T^{n} ; W\right) \cap L^{2}\left(0, T^{n} ; V\right) \cap H^{1}\left(0, T^{n} ; V^{\prime}\right), \\
w_{m}^{n} \rightarrow w^{n} \quad(\forall \delta>0) \\
\text { weakly star in } L^{\infty}\left(0, T^{n} ; W\right) \cap L^{2}\left(0, T^{n} ; V\right) \cap H^{1 / 2-\delta}\left(0, T^{n} ; W\right) \\
\chi_{m}^{n} \rightarrow \chi^{n} \quad \text { weakly star in } W^{1, \infty}\left(0, T^{n} ; L^{\infty}(\Omega)\right), \\
\beta\left(\theta_{m}^{n}, w_{m}^{n}\right) \rightarrow \xi^{n} \quad \text { weakly star in } L^{\infty}\left(Q_{n}\right) .
\end{gathered}
$$

By Aubin's lemma (cf. [17, p.57]), (4.25) and (4.26) yield

$$
\begin{array}{ccc}
\theta_{m}^{n} \rightarrow \theta^{n} & \text { strongly in } L^{2}\left(0, T^{n} ; H^{1-\delta}(\Omega)\right), & \forall \delta>0, \\
w_{m}^{n} \rightarrow w^{n} & \text { strongly in } L^{2}\left(0, T^{n} ; H^{1-\delta}(\Omega)\right), & \forall \delta>0 ;
\end{array}
$$

hence

$$
\begin{gathered}
b_{m}^{n}\left(w_{m}^{n}\right) \rightarrow b^{n}\left(w^{n}\right) \text { strongly in } L^{2}\left(0, T^{n} ; H^{1-\delta}(\Omega)\right), \forall \delta>0, \\
\xi^{n}=\beta\left(\theta^{n}, w^{n}\right) \text { a.e. in } Q^{n} .
\end{gathered}
$$

We indicate by $(\mathrm{P} 2)^{n}$ the problem obtained by replacing $-w^{-}$with $b^{n}(w)$ in (P2); taking $m \rightarrow \infty$ in $(\mathrm{P} 2)_{m}^{n}$ we get that $\left(\theta^{n}, w^{n}, \chi^{n}\right)$ solves $(\mathrm{P} 2)^{n}$.

(iv) Limit as $n \rightarrow \infty$.

In order to deduce a priori estimates uniform in $n$, we multiply the $\theta^{n}$-equation of (P2) ${ }^{n}$ by $\theta^{n}$, the $w^{n}$-equation by $w^{n}$ and the $\chi^{n}$-equation by $\partial \chi^{n} / \partial t$, sum them and integrate in time. We notice that by (4.9), (4.5) (now written for $\chi^{n}$ ) yields

$$
\left.\left\|\frac{\partial \chi^{n}}{\partial t}\right\|_{L^{\infty}\left(Q^{n}\right)} \leqslant \text { Constant (independent of } n\right) \text {; }
$$


hence, setting $B^{n}(\xi):=\int_{0}^{\xi} b^{n^{\prime}}(\eta) \eta d \eta, \forall \xi \in \mathbf{R}$, we have (cf. with (4.20))

$$
\begin{aligned}
\int_{\Omega} \frac{\partial}{\partial t}\left[b^{n}\left(w^{n}\right) \cdot \chi^{n}\right] w^{n} d x= & \frac{1}{2} \frac{d}{d t} \int_{\Omega} \chi^{n} \cdot B^{n}\left(w^{n}\right) d x \\
& -\frac{1}{2} \int_{\Omega} \frac{\partial \chi^{n}}{\partial t} \cdot B^{n}\left(w^{n}\right) d x+\int_{\Omega} b^{n}\left(w^{n}\right) \cdot \frac{\partial \chi^{n}}{\partial t} \cdot w^{n} d x \\
\geqslant & \frac{1}{2} \frac{d}{d t} \int_{\Omega} \chi^{n} \cdot B^{n}\left(w^{n}\right) d x-\frac{5}{4}\left\|\frac{\partial \chi^{n}}{\partial t}\right\|_{L^{\infty}\left(Q^{n}\right)} \cdot\left\|\left(w^{n}\right)^{-}\right\|_{w}^{2},
\end{aligned}
$$

with constants independent of $n$. This makes it possible to prove for $\theta^{n}, w^{n}, \chi^{n}$ estimates of the type of $(4.21), \ldots,(4.23)$ uniform in $n$. Finally, possibly extracting subsequences, we get the convergence to a solution of (P2).

Maximum and minimum principles can be proved, as in Sec. 3. For instance:

Proposition 3 (Maximum and minimum principles for $w$ ). Let $N \in \mathbf{R}^{+}$. Assume that (4.1), (4.2) hold and that

$$
\begin{gathered}
\beta(\xi, \eta) \geqslant 0 \quad \forall \xi \in \mathbf{R}, \quad \forall \eta \in[0, N], \\
\left\{\begin{array}{c}
\text { there exist } \chi^{0} \in L^{\infty}(\Omega ;[0,1]) \text { and } w^{0} \in W \text { such that } \\
\omega^{0}=\gamma_{0} w^{0}-l \cdot\left(w^{0}\right)^{-} \cdot \chi^{0} \text { a.e. in } \Omega ;\left|w^{0}\right| \leqslant N \text { a. e. in } \Omega, \\
f_{2} \leqslant 0 .
\end{array}\right.
\end{gathered}
$$

Then for any solution of problem (P2)

$$
|w| \leqslant N \text { a.e. in } Q \text {. }
$$

Proof. We multiply (4.4) by $\min [\max (w,-N), N]$.

Here we can also prove a uniqueness result:

Theorem 5. Assume that (4.1), (4.2), and the assumptions of Proposition 3 hold, and that

$$
\beta \text { is Lipschitz continuous (with constant } \Lambda \text { ). }
$$

Then problem (P2) has at most one solution.

Proof. Let $\left(\theta_{i}, w_{i}, \chi_{i}\right)(i=1,2)$ be two solutions of (P2). We set $\tilde{\theta}:=\theta_{1}-\theta_{2}, \tilde{w}:=w_{1}$ $w_{2}, \tilde{\chi}:=\chi_{1}-\chi_{2}$ a.e. in $Q$. We write (4.3), (4.4), and (4.5) for $i=1,2$ and take the differences; then we integrate the first two in time, multiply the three of them by $\tilde{\theta}, \tilde{w}, \tilde{\chi}$, respectively, and sum the resulting expressions. Then, since

$$
-l w_{1}^{-} \chi_{1}+l w_{2}^{-} \chi_{2}=-l\left(w_{1}^{-}-w_{2}^{-}\right) \chi_{1}-l w_{2}^{-} \tilde{\chi} \geqslant-l|\tilde{w}| \chi_{1}-l w_{2}^{-} \tilde{\chi},
$$

we get

$$
\begin{aligned}
& \int_{\Omega}\left[\gamma_{1} \tilde{\theta}^{2}+\left(\gamma_{0}-l \chi_{1}\right) \tilde{w}^{2}\right] d x+\frac{1}{2} \frac{d}{d t}\left(\int_{\Omega} \tilde{\chi}^{2} d x+{ }_{V^{\prime}}\left\langle A_{1} \int_{0}^{t} \tilde{\theta} d \tau, \int_{0}^{t} \tilde{\theta} d \tau\right\rangle_{V}\right. \\
& \left.\quad+{ }_{V^{\prime}}\left\langle A_{2} \int_{0}^{t} \tilde{w} d \tau, \int_{0}^{t} \tilde{w} d \tau\right\rangle_{V}\right) \leqslant L\|\tilde{\chi}\|_{L^{2}(\Omega)} \cdot\|\tilde{\theta}\|_{L^{2}(\Omega)} \\
& +l\left\|w_{2}^{-}\right\|_{L^{\infty}(Q)} \cdot\|\tilde{\chi}\|_{L^{2}(\Omega)} \cdot\|\tilde{w}\|_{L^{2}(\Omega)}+\Lambda\left(\|\tilde{\theta}\|_{L^{2}(\Omega)}+\|\tilde{w}\|_{L^{2}(\Omega)}\right) \cdot\|\tilde{\chi}\|_{L^{2}(\Omega)}
\end{aligned}
$$


We recall that $\gamma_{0}>l$ : moreover, by Proposition $3,\left\|w_{2}^{-}\right\|_{L^{\infty}(Q)} \leqslant N$. Then by Gronwall's lemma we get $\tilde{\theta}=\tilde{w}=\tilde{\chi}=0$ a.e. in $Q$.

Remark. The Lipschitz-continuous dependence on the data can be proved similarly.

Finally we state two regularity results:

TheOrem 6. Assume that (4.1), (4.2), (4.9), (4.34) hold and that

$$
\begin{gathered}
\theta^{0}, \chi^{0} \in V, \\
f_{i}=f_{i}^{(1)}+f_{i}^{(2)}, \quad f_{i}^{(1)} \in L^{2}(Q), \quad f_{i}^{(2)} \in W^{1,1}\left(0, T ; V^{\prime}\right) \quad(i=1,2) .
\end{gathered}
$$

Then problem (P2) has at least one solution such that

$$
\theta, w \in H^{1}(0, T ; W) \cap L^{\infty}(0, T ; V) ; \chi \in W^{1, \infty}\left(0, T ; L^{\infty}(\Omega)\right) .
$$

Outline of the proof. We take $v=\partial \theta_{m}^{n} / \partial t$ in (4.14), $v=\partial w_{m}^{n} / \partial t$ in (4.15), then derive (4.16) in time and multiply it by $\partial \chi_{m}^{n} / \partial t$; by summing these equations and integrating in time, we obtain a priori estimates corresponding to (4.38).

TheOrem 7. Assume that (4.1), (4.2), (4.9), (4.34) hold and that

$$
\chi^{0} \in V \text {. }
$$

Then problem (P2) has at least one solution such that

$$
\chi \in L^{\infty}(0, T ; V) \text {. }
$$

Outline of the proof. For any $m \in \underline{\mathbf{N}}$, let $\Psi_{m}$ be as in the proof of Theorem 4 and let $\left(\theta_{m}, w_{m}, \chi_{m}\right)$ be a solution of problem $(\widetilde{\mathrm{P} 3})_{m}$, obtained by replacing $(4.5)$ with

$$
\frac{\partial \chi_{m}}{\partial t}+\Psi_{m}\left(\chi_{m}\right)=\beta\left(\theta_{m}, w_{m}\right) \text { a.e. in } Q .
$$

Now we apply the space gradient to the latter equation; assuming for simplicity that $\beta$ be differentiable, we get

$$
\frac{\partial \bar{\nabla} \chi_{m}}{\partial t}+\Psi_{m}^{\prime}\left(\chi_{m}\right) \bar{\nabla} \chi_{m}=\frac{\partial \beta}{\partial \theta}\left(\theta_{m}, w_{m}\right) \cdot \bar{\nabla} \theta_{m}+\frac{\partial \beta}{\partial w}\left(\theta_{m}, w_{m}\right) \cdot \bar{\nabla} w_{m} .
$$

Then we multiply this by $\bar{\nabla} \chi_{m}$ and integrate in space and time. This procedure is just formal, but it can be made rigorous by using an approximation technique which yields more regularity for $\chi_{m}$, or also by replacing the gradients by the incremental ratios in space. By (4.34) we get

$$
\begin{aligned}
\frac{1}{2}\left\|\bar{\nabla} \chi_{m}(t)\right\|_{L^{2}(\Omega)^{3}}^{2} & -\frac{1}{2}\left\|\bar{\nabla} \chi^{0}\right\|_{L^{2}(\Omega)^{3}}^{2} \\
& \leqslant \Lambda\left(\left\|\bar{\nabla} \theta_{m}\right\|_{L^{2}(Q)^{N}}+\left\|\bar{\nabla} w_{m}\right\|_{L^{2}(Q)^{N}}\right) \cdot\left(\int_{0}^{t}\left\|\bar{\nabla} \chi_{m}(x, t)\right\|_{L^{2}(\Omega)^{N}}^{2} d t\right)^{1 / 2},
\end{aligned}
$$

whence an a priori estimate corresponding to (4.40).

Generalizations. (i) The existence and regularity results of this section can be easily extended to the case of $\gamma_{1}=\hat{\gamma}_{1}(\theta), \gamma_{0}=\hat{\gamma}_{0}(w), \ell=\hat{\ell}(w)$, under natural assumptions for the given functions $\hat{\gamma}_{1}, \hat{\gamma}_{2}, \hat{\ell}$. As for the uniqueness result, we notice that

$$
\left[\hat{\gamma}_{1}\left(\theta_{1}\right) \theta_{1}-\hat{\gamma}_{1}\left(\theta_{2}\right) \theta_{2}\right] \cdot\left(\theta_{1}-\theta_{2}\right) \geqslant\left(\min \hat{\gamma}_{1}-\frac{\left|\hat{\gamma}_{1}\left(\theta_{1}\right)-\hat{\gamma}_{1}\left(\theta_{2}\right)\right|}{\left|\theta_{1}-\theta_{2}\right|}\left|\theta_{1}\right|\right) \cdot\left(\theta_{1}-\theta_{2}\right)^{2}
$$


hence also Theorem 5 can be extended, if maximum and minimum principles hold for $\theta$ and if $\hat{\gamma}_{1}$ is Lipschitz-continuous with Lipschitz-constant $\tilde{\Lambda}<\|\theta\|_{I^{\infty}(Q)}^{-1} \cdot \min \hat{\gamma}_{1}$.

(ii) The case of phase-dependent specific heat and temperature-dependent latent heat can be treated as for problem (P1).

(iii) An existence result can be proved in the case of nonconstant and phase-dependent conductivities, namely $k_{i}=\hat{k}_{i}(\theta, w, \chi)(i=1,2)$, thanks to the regularity property (4.40).

(iv) In the case of vanishing mass conductivity in the solid phase, even the existence of a weak solution is an open question.

(v) Finally we consider the case in which the phase transition velocity depends also on $\chi$, i.e., (4.5) is substituted with

$$
\frac{\partial \chi}{\partial t}+\tilde{H}^{-1}(\chi) \ni \beta(\theta, w, \chi) \text { a.e. in } Q .
$$

For instance

$$
\beta(\theta, w, \chi)=\mu_{1}(\theta-w)^{+}(1-\chi)-\mu_{2}(\theta-w)^{-} \chi\left(\mu_{1}, \mu_{2} \in \mathbf{R}^{+}\right) .
$$

In this case, for fixed $\theta-w, \chi$ has an exponential decay in time. In general $\beta$ is antimonotone in $\chi$; hence $-\beta(\theta, w, \chi)=(\partial \Psi / \partial \chi)(\theta, w, \chi)$, with $\Psi$ continuous in $\theta, w$ and convex in $\chi$. Thus (4.41) corresponds to the following variational inequality:

$$
\left\{\begin{array}{l}
\text { a.e. in } Q, 0 \leqslant \chi \leqslant 1 \text { and } \forall v: \Omega \rightarrow[0,1] \text { measurable, } \\
\left.\int_{\Omega} \frac{\partial \chi}{\partial t}(\chi-v) d x+\int_{\Omega}[\Psi(\theta, w, \chi)-\Psi(\theta, w, v)] d x \leqslant 0, \text { a.e. in }\right] 0, T[.
\end{array}\right.
$$

The results of this section can be easily extended here. A priori estimates can be proved as above; the continuity of $\Psi$ in $\theta, w$ and its convexity in $\chi$ make it possible to take the limit in the approximated (4.43). Also uniqueness holds, if $\beta$ is Lipschitz-continuous in $\theta, w$, uniformly with respect to $\chi$ (as in (4.42), e.g.).

\section{REFERENCES}

[1] G. Astarita and G. C. Sarti, A class of mathematical models for sorption of swelling solvents in glassy polymers, Polymer Eng. Science 18, 388-395 (1978)

[2] A. Bermudez and C. Saguez, Mathematical formulation and numerical solution of an alloy solidification problem in [12]

[3] B. Boley, An applied overview of moving boundaries, in [31]

[4] J. R. Cannon, J. C. Cavendish and A. Fasano, A free boundary-value problem related to the combustion of a solid, SIAM J. Appl. Math. 45, 798-809 (1985)

[5] B. Chalmers, Principles of solidification, Krieger, New York (1977)

[6] J. Crank, Free and moving boundary problems, Clarendon Press, Oxford (1984)

[7] A. B. Crowley, Numerical solution of alloy solidification problems revisited, in A. Bossavit, A. Damlamian, M. Fremond (editors), Free boundary problems: applications and theory, vols. III, IV, Pitman, London (1985)

[8] A. B. Crowley and J. R. Ockendon, On the numerical solution of an ulloy solidification problem. Internat. J. Heat Mass Transfer 22, 941-946 (1979)

[9] J. D. P. Donnelly, A model for non-equilibrium thermodynamic processes involving phase changes, J. Inst. Math. Appl. 24, 425-438 (1979)

[10] C. M. Elliott and J. R. Ockendon, Weak and variational methods for moving boundary problems, Pitman, London (1982)

[11] A. Fasano, C. Meyer, and M. Primicerio, On a problem in polymer industry: theoretical and numerical investigation, to appear in SIAM J. 
[12] A. Fasano and M. Primicerio (editors), Free boundary problems: theory and applications, vols. I, II, Pitman, London (1983)

[13] G. J. Fix, Numerical methods for alloy solidification problems in [31]

[14] G. J. Fix, Phase field models for free boundary problems, in [12]

[15] M. C. Flemings, Solidification processing, McGraw-Hill, New York (1974)

[16] M. Fremond and A. Visintin, Dissipation dans le changement de phase. Surfusion. Changement de phase irréversible, C. R. Acad. Sci. Paris 301, 1265-1268 (1985)

[17] J. L. Lions, Quelques méthodes de résolution des problèmes aux limites non lineaires, Dunod, Paris (1969)

[18] S. Luckhaus and A. Visintin, Phase transition in multicomponent systems, Manuscripta Math. 43, 261-288 (1983)

[19] E. Magenes (editor), Free boundary problems, Proceedings of a congress held in Pavia, Sept.-Oct. 1979, Ist. Naz. Alta Matem., Roma (1980)

[20] J. R. Ockendon and W. R. Hodgkins (editors), Moving boundary problems in heat flow and diffusion, Clarendon Press, Oxford (1975)

[21] L. I. Rubinstein, The Stefan problem, Translations of Mathematical Monographs 27, Amer. Math. Soc., Providence (1971)

[22] H. Triebel, Interpolation theory, function spaces, differential operators, North-Holland, Amsterdam (1978)

[23] C. Verdi and A. Visintin, Numerical analysis of the multidimensional Stefan problem with supercooling and superheating, to appear in Boll. Un. Mat. Ital.

[24] A. Visintin, Stefan problem with phase relaxation, I.M.A. J. Appl. Math. 34, 225-245 (1985)

[25] A. Visintin, Stefan problem with surface tension, Preprint of I. A. N. of C. N. R. (1984)

[26] A. Visintin, Models for supercooling and superheating effects, in A. Bossavit, A. Damlamian, M. Fremond (editors), Free boundary problems: applications and theory, vols. III, IV, Pitman, London (1985)

[27] A. Visintin, Supercooling and superheating effects in phase transitions, I.M.A. J. Appl. Math. 35, 233-256 (1985)

[28] A. Visintin, Stefan problem with a kinetic condition at the free boundary, Preprint of I.A.N. of C.N.R. (1985), to appear in Ann. Mat. Pura e Appl.

[29] A. Visintin, Coupled termic and electromagnetic evolution in a ferromagnetic body, to appear in Z.A.M.M.

[30] R. E. White, The binary alloy problem: existence, uniqueness and numerical approximation, SIAM J. Numer. Anal. 22, 205-244 (1985)

[31] D. G. Wilson, A. D. Solomon, and P. T. Boggs (editors), Moving boundary problems, Academic Press, New York (1978) 